\title{
The LINC00504/Mir-4739/KLK4 Axis Facilitates Cell Proliferation and Suppresses Cell Apoptosis Through Triggering The Wnt/B-Catenin Pathway In Triple-Negative Breast Cancer Cells
}

\section{Di Wu}

The First Hospital of Jilin University

Hongyao Jia

The First Hospital of Jilin University

Zhiru Zhang

The First Hospital of Jilin University

Sijie Li ( $\square$ lisij@jlu.edu.cn )

The First Hospital of Jilin University

\section{Research article}

Keywords: triple-negative breast cancer, LINC00504, Wnt/ $\beta$-catenin pathway, miR-4739, KLK4

Posted Date: December 1st, 2020

DOI: https://doi.org/10.21203/rs.3.rs-115872/v1

License: (c) (1) This work is licensed under a Creative Commons Attribution 4.0 International License.

Read Full License 


\section{Abstract}

Background: Currently, long non-coding RNAs (IncRNAs) have been validated to exert critical influence on the malignant progression of triple-negative breast cancer (TNBC). LncRNA long intergenic non-protein coding RNA 504 (LINC00504) has been recently reported as a tumor facilitator in the cellular processes of several cancers. However, its function in TNBC remains unknown.

Methods: CCK-8 and colony formation assays were used to detect the cell viability and proliferation in TNBC. Flow cytometry analysis was utilized to measure the cycle and apoptosis of TNBC cells. The levels of key proteins associated with cell apoptosis or the $\beta$-catenin pathway were detected through western blot analysis. The activity of the Wnt/ $\beta$-catenin signaling pathway was measured by the TOP/FOP flash assay. ChIP assay was conducted to confirm the binding between LINC00504 and its transcription factor signal transducer and activator of transcription 3 (STAT3). RIP and luciferase reporter assays were used to detect and verify the interaction among LINC00504 and its downstream molecule.

Results: LncRNA LINC00504 was upregulated in TNBC, and silenced LINC00504 suppressed cell proliferation and triggers cell cycle arrest at G0/G1 stage and cell apoptosis in TNBC cells. STAT3 can transcriptionally activate LINC00504 and LINC00504 served as a molecular sponge of microRNA (miR4379). Kallikrein related peptidase 4 (KLK4) was the target gene of miR-4379 and activates the Wnt/ $\beta$ catenin pathway. LINC00504 upregulated KLK4 via competitively binding with miR-4379 to activate the Wnt/ $\beta$-catenin pathway in TNBC. The suppression on TNBC cell proliferation and the promotion on TNBC cell cycle arrest and apoptosis under LINC00504 knockdown were rescued by miR-4379 depletion or KLK4 overexpression.

Conclusions: The LINC00504/miR-4379/KLK4 axis promotes cell growth and cell cycle progression as well as suppresses cell apoptosis through activating the $\mathrm{Wnt} / \beta$-catenin pathway.

\section{Background}

As a prevalent cancer among females globally, breast cancer seriously threatens the health of women [1]. Breast cancer is a dominant disease in gynecological oncology [2]. Triple-negative breast cancer (TNBC) is a particularly aggressive subtype of breast cancer characterized by the worst prognosis [3]. The definition of TNBC is adaptive to all tumors lacking the expression of ER, PR and HER2, which are all molecular targets for therapy [4]. Over the past few years, great progresses have been made in improving clinical therapeutics for breast cancer. However, now encouraging clinical activity is seen in the breast oncology community from molecularly targeted approaches to TNBC [5]. Thus, it is meaningful to figure out novel molecule targets for TNBC.

Long non-coding RNAs (IncRNAs) have over 200 nucleotides with limited or without coding ability. LncRNAs play pivotal regulatory roles in cell malignant behaviors including cell proliferation, migration, invasion and apoptosis [6, 7]. Recently, accumulating reports have revealed that IncRNAs act as tumor facilitators and suppressors, thereby affecting the development of tumors $[8,9]$. For example, long non- 
coding RNA MNX1-AS1 promotes TNBC development by enhancing phosphorylation of Stat3 [10]. PCAT6 promotes TNBC cell proliferation, migration, invasion, and EMT [11]. Upregulation of GATA3-AS1 contributes to TNBC cell proliferation and migration [12]. The role of IncRNA long intergenic non-protein coding RNA 504 (LINC00504) has been identified in several cancers. LINC00504 acts as a therapeutic biomarker for patients with non-small cell lung cancer [13]. LINC00504 promotes ovarian cancer cell development and stimulates aerobic glycolysis [14]. LINC00504 functions as a critical transcriptional regulator for C-Myc in cells of colon cancer [15]. Based on the Gene Expression Profiling Interactive Analysis (GEPIA) database (http://gepia.cancer-pku.cn/), we found that LINC00504 was highly expressed in breast cancer. Therefore, we aimed to figure out the function of LINC00504 in TNBC.

Many researches have indicated that IncRNAs act as competing endogenous RNAs (ceRNAs) by sponging microRNAs (miRNAs) and then modulate expression of target messenger RNAs (mRNAs) [16, 17]. Currently, ceRNA networks have been confirmed in progression of TNBC. For instance, IncRNA LRRC75A-AS1 acts as a ceRNA that promotes proliferation, invasion and EMT process of TNBC cells through the miR-380-3p/BAALC axis [18]. LncRNA HOST2 facilitates STAT3-mediated cell proliferation and migration as a ceRNA to decoy let-7b in TNBC [19]. LncRNA ARNILA serves as a ceRNA for miR-204 to upregulate Sox4, accelerating EMT, invasion and metastasis of TNBC [20]. Therefore, we planned to probe the functional meaning of LINC00504 and the putative ceRNA pattern mediated by LINC00504 in TNBC cells.

\section{Methods}

\subsection{Clinical samples}

Thirty-two pairs of TNBC tissues and adjacent non-tumor tissues were collected from TNBC patients. No patients had received chemotherapy or radiotherapy formerly at the First Hospital of Jilin University (Jilin, China). This research was approved by the Ethics Committee of the First Hospital of Jilin University (Jilin, China). All patients signed the informed consents in written form.

\subsection{Cell culture}

The TNBC cell lines (SKBR-3, MDA-MB-231 and BT-549) and the normal breast cell line (MCF-10A) were purchased from American Type Culture Collection (ATCC, USA) and were inoculated in Roswell Park Memorial Institute 1640 (RPMI 1640) medium (ThermoFisher, Waltham, MA, USA) including 10\% fetal bovine serum (FBS, ThermoFisher), $100 \mathrm{mg} / \mathrm{mL}$ of streptomycin (ThermoFisher) and $100 \mathrm{U} / \mathrm{mL}$ of penicillin (ThermoFisher) at $37^{\circ} \mathrm{C}$ in $5 \% \mathrm{CO}_{2}$.

\subsection{Cell transfection}

The sh-LINC00504\#1/2 and negative control (sh-NC) were bought from OriGene Technologies (USA). The overexpression of kallikrein related peptidase 4 (KLK4) was provided by OriGene Technologies. MicroRNA 4739 (miR-4739) inhibitor, miR-4739 mimics, and their corresponding negative controls (NC-inhibitor and NC-mimics) were obtained from GenePharma (Shanghai, China). The lipofectamine 2000 (Invitrogen, 
USA) was applied to achieve transfection. After incubated $4 \mathrm{~h}$ at $37^{\circ} \mathrm{C}$, the fresh RPMI 1640 medium with $10 \% \mathrm{FBS}$ was applied to replace the medium. After being incubated $48 \mathrm{~h}$ at $37^{\circ} \mathrm{C}$, the cells were collected for further analysis.

\subsection{Reverse transcription quantitative polymerase chain reaction (RT-qPCR)}

The TRIzol (Life technologies, New York, NY, USA) kit was utilized to extract RNA, and the Prime Script RT reagent Kit (TaKaRa, Dalian, China) was used for reverse transcription. Next, the SYBR ${ }^{\circledR}$ Premix Ex Taq ${ }^{\text {TM }}$ (TaKaRa) was utilized to conduct RT-qPCR analyses in the ABI Prism 7500 Fast Real-Time PCR system (Applied Biosystems, CA, USA). Later the Genechem Co., Ltd. (Shanghai, China) synthesized the primer sequences for LINC00504, miR-4739 and KLK4. Glyceraldehyde phosphate dehydrogenase (GAPDH) functioned as the internal reference for LINC00504 and KLK4, and RNU6 (U6) acted as the internal reference for miR-4739. Relative expression of RNAs was calculated using $2^{-\Delta \Delta C t}$ method.

\subsection{Cell counting kit-8 (CCK-8) assay}

Cells (with density of $3 \times 10^{3}$ cells/well) were incubated in a 96-well plate. CCK-8 solution ( $10 \mu \mathrm{L}$, SigmaAldrich, St. Louis, MO, USA) was added into each well and then the cells were cultured for $2 \mathrm{~h}$. At $0,24,48$, $72 \mathrm{~h}$, cell viability was measured and qualified by the optical density value at $450 \mathrm{~nm}$ by a microplate reader (Molecular Devices, San Francisco, CA, USA).

\subsection{Colony formation assay}

SKBR-3 and MDA-MB-231 cells were seeded in a six-well plate, cultured with $10 \%$ FBS and preserved in RPMI 1640 medium for 10 days. Methanol was applied to fix colonies for $15 \mathrm{~min}$ at ambient temperature. After that, crystal violet dye was used to stain cells for $15 \mathrm{~min}$. The Image $\mathrm{J}$ software was used to assess number of colonies ( $>50$ cells).

\subsection{Cell cycle analysis}

The cell cycle was measured in SKBR-3 and MDA-MB-231 cells using flow cytometry. The propidium iodide (PI) (Sigma-Aldrich) was applied to harvest and stain cells. After washing the cells by phosphate buffer saline (PBS) twice and centrifuging them, the cell suspension was fixed at chilled $70 \%$ ethanol and incubated $4^{\circ} \mathrm{C}$ overnight. Next, $100 \mu \mathrm{g} / \mathrm{ml}$ of RNase A was added, and the mixture was cultured at $37^{\circ} \mathrm{C}$ for $30 \mathrm{~min}$. After adding PI solution, the mixture was incubated at $4^{\circ} \mathrm{C}$ for $30 \mathrm{~min}$ in the darkness. Finally, a FACSCalibur flow cytometry (BD Biosciences, Franklin Lake, NJ, USA) and the ModiFitLT software were used to analyze samples.

\subsection{Cell apoptosis}

Cells $\left(5 \times 10^{4}\right.$ cells/well) were grown in six-well plate and cultured overnight. After treatment, cold PBS was re-suspended in binding buffer (BD Biosciences) and were applied to wash the harvested cells twice. Cells were dyed using $5 \mu$ l of Annexin V- Fluorescein Isothiocyanate (FITC) and $5 \mu$ l of Pl, lightly mixed 
and reacted in conditions devoid of light for 10-15 min at room temperature. Eventually, a FACSCalibur flow cytometer (BD Biosciences) was used to analyze the cell apoptosis.

\subsection{Western blot}

Ice-cold PBS was used to collect and wash the cells twice. Proteins were extracted with RNA immunoprecipitation assay (RIPA) lysis buffer. After being centrifuged and heated, the electrophoresis in $12 \%$ sodium dodecyl sulfate, polyacrylamide gel electrophoresis (SDS-PAGE) was carried out to fractionate lysates. Protein lysates were transferred to polyvinylidene difluoride (PVDF) membranes and closed by $5 \%$ skim milk for $2 \mathrm{~h}$ at ambient temperature. Later, these membranes were cultured with primary antibodies including: anti-Bax (ab182733, 1:1000, Abcam, Cambridge, UK), anti-Bcl-2 (ab182858, 1:2000, Abcam), anti- $\beta$-catenin (ab32572, 1:5000, Abcam), anti-c-Myc (ab32072, 1:1000, Abcam). The mixture was then incubated overnight at $4^{\circ} \mathrm{C}$ with continuous agitation. Secondary antibodies were used to culture the membranes for $2 \mathrm{~h}$ at $37^{\circ} \mathrm{C}$. The Tris Buffered Saline Tween (TBST) was used to wash the membranes for three times. The ImageJ software was used to detect densitometry analysis of the band intensity.

\subsection{Caspase-3 activity detection}

The relative caspase-3 activity was measured by the Caspase-3 Colorimetric Assay Kit (Keygen, Jiangsu, China) in line with the manufacturer's protocols.

\subsection{Chromatin immunoprecipitation (ChIP) assay}

The crosslinked chromatin was sheared and immunoprecipitated with anti-STAT3 antibody (ab68153, Abcam) or anti-lgG antibody (ab6789, Abcam) as control, along with the magnetic beads. At last, precipitates were purified for RT-qPCR.

\subsection{Luciferase reporter assay}

The sequence of LINC00504 promoter was subcloned into the luciferase reporter pGL3-vector (Promega, Madison, WI, USA). LINC00504-WT/Mut pmirGLO vectors or KLK4 3'untranslated region (UTR)-WT/Mut pmirGLO vectors (GenePharma, Shanghai, China) were constructed and transfected into SKBR-3 and MDA-MB-231 cells with miR-4739 mimics, NC-mimics, miR-4739 inhibitor or NC-inhibitor. $48 \mathrm{~h}$ after transfection, the luciferase activity was determined by the Dual-Luciferase Reporter Assay System (Promega, USA).

\subsection{RNA immunoprecipitation (RIP) assay}

A Magna RIP RNA Binding Protein Immunoprecipitation Kit (Millipore, Danvers, MA, USA) was utilized to perform RIP assay. Next, precooled PBS was utilized to wash cells, and the supernatant was taken out. 
After the cells were lysed and centrifuged, the supernatant was discarded. The RIPA buffer was used to resuspend the magnetic beads-antibody complex incubated at $4{ }^{\circ} \mathrm{C}$. Next, the magnetic bead-protein complex was collected via the sample placed on the magnetic pedestal. The antibodies were anti-Ago2 (ab 186733, Abcam), and anti-IgG (ab6715, Abcam) served as the negative control (NC). The endogenous miR-4739 that combined with LINC00504/KLK4 was precipitated. Subsequently, cell lysis in the RIPA buffer was incubated with magnetic bead conjugated with human Ago2 antibody (Millipore, USA). The purified RNA concentration was determined using RT-qPCR analysis.

\subsection{TOP/FOP flash assay}

The TOP or FOP plasmids were co-transfected with expression regulation plasmids into TNBC cells. After $48 \mathrm{~h}$ of incubation, a Dual Luciferase Reporter Assay Kit (Promega) was used to measure the activity of the Wnt/ $\beta$-catenin signaling pathway.

\subsection{Subcellular fraction assay}

The nuclear and cytoplasmic LINC00504 were isolated by the PARIS ${ }^{\text {TM }}$ kit (Thermo Fisher Scientific). LINC00504 from isolated nuclear and cytoplasmic fractions was reversely transcribed and applied for PCR analysis after purification. U6 was used as the internal reference for the nucleus, and GAPDH as the internal reference for the cytoplasm.

\subsection{Statistical Analysis}

SPSS 21.0 (SPSS, USA) software was utilized to conduct the data analysis. We used mean \pm standard deviation (SD) to exhibit the obtained data. The Student's t-test was applied for comparisons of two groups, and one-way/two-way analysis of variance was utilized for comparisons among different groups, and then a Tukey's post hoc test was conducted. Each experiment was repeated at least three times. $p<$ 0.05 was considered as significantly statistical.

\section{Results}

\subsection{LncRNA LINC00504 is upregulated in TNBC tissues and cells}

LncRNA LINC00504 has been indicated as an oncogene to promote progression of many cancers. Here, we explored the expression level of LINC00504 in TNBC. The overexpression of LINC00504 in breast cancer tissues was revealed from the database of GEPIA (Fig. 1A). The results from RT-qPCR showed that LINC00504 was highly expressed in TNBC cell lines, in comparison to control cell line (Fig. 1B). Furthermore, we verified that LINC00504 was overexpressed in TNBC tissues in contrast with adjacent 
noncancerous tissues (Fig. 1C). After that, it was confirmed that LINC00504 expression was higher in advanced-stage tissues than in early-stage tissues (Fig. 1D). All these data revealed that LINC00504 was upregulated in TNBC cell lines and tissues.

\subsection{Downregulation of LINC00504 suppresses cell proliferation and triggers cell cycle arrest and cell apoptosis in TNBC cells}

Next, shRNA-LINC00504 plasmids were transfected into TNBC cells, and the knockdown efficiency of LINC00504 was verified through RT-qPCR analysis (Fig. 2A). Cell viability of SKBR-3 and MDA-MB-231 cells after silencing LINC00504 was accessed via CCK-8 assay. It was suggested that LINC00504 knockdown inhibited cell viability (Fig. 2B). In addition, the effects of sh-LINC00504\#1/2 on cell proliferation was assessed. As revealed in Fig. 2C, inhibition of LINC00504 reduced the number of colonies in colony formation assay. Additionally, we studied the influence of LINC00504 silencing on cell cycle through flow cytometry. The LINC00504 knockdown elevated the percentage of SKBR-3 and MDAMB-231 cells in the G0/G1 phase and declined that in the S phase, suggesting that LINC00504 knockdown can trigger cell cycle arrest at G0/G1 phase (Fig. 2D). Moreover, we detected cell apoptosis in TNBC cells using flow cytometry. After silencing LINC00504, cell apoptotic rate was increased (Fig. 2E). Additionally, we detected the activity of caspase-3, and found that the activity of caspase-3 was significantly increased by silencing of LINC00504 (Fig. 2F). We conducted the western blot to determine the expression of apoptosis-related proteins (Bax and Bcl-2) in SKBR-3 and MDA-MB-231 cells. Results depicted that Bax level was elevated and Bcl-2 expression was repressed after silencing LINC00504 (Fig. 2G). These findings showed that LINC00504 downregulation repressed cell growth and induced cell apoptosis and cell cycle arrest in the G0/G1 phase in TNBC cells.

\subsection{LINC00504 is transcriptionally activated by STAT3}

After that, we sought to examine the upstream transcription factor for LINC00504. As was predicted from UCSC, STAT3 is putative to serve as the transcription factor for LINC00504. We effectively overexpressed expression of STAT3 via transfection of pcDNA3.1-STAT3 and validated that up-regulation of STAT3 significantly increased expression of LINC00504 (Fig. 3A). Spearman's correlation analysis from the GEPIA database suggested that there was a positive relationship between LINC00504 expression and STAT3 expression in TNBC tissues (Fig. 3B). Next, through RT-qPCR, the expression of STAT3 was revealed to be upregulated in TNBC tissues than adjacent normal tissues (Fig. 3C). In addition, STAT3 was confirmed to be highly expressed in TNBC cell lines than control cell line (Fig. 3D). Then, the DNA motif of STAT3 was predicted from JASPAR website (http://jaspar.genereg.net/matrix/MA0079.1/) (Fig. 3E). ChIP assay showed that anti-STAT3 significantly precipitated LINC00504 promoter in TNBC 
cells (Fig. 3F). As was shown in Fig. 3G, overexpression of STAT3 increased the luciferase activity of pGL3 vectors containing LINC00504 promoter in TNBC cells. To conclude, STAT3 served as the transcription factor for LINC00504.

\subsection{LINC00504 serves as a molecular sponge of miR-4739}

Subsequently, we aimed to explore the potential mechanism underlying LINC00504 on TNBC cell malignant behaviors. It was demonstrated that IncRNAs act as ceRNAs to sponge miRNAs at the posttranscriptional level, thereby regulating cancer tumorigenesis $[16,17]$. Thus, we detected the subcellular location of LINC00504 through subcellular fraction assay. As was revealed in Fig. 4A, LINC00504 was mainly located in the cytoplasm of SKBR-3 and MDA-MB-231 cells. Then, we predicted the potential miRNAs binding with LINC00504 by usage of DIANA tool (http://diana.imis.athenainnovation.gr/DianaTools/index.php?r=site/index). Among 5 candidate miRNAs, only miR-4739 was under-expressed in TNBC cells (Fig. 4B). Then through RT-qPCR analysis, miR-4739 was revealed to be downregulated in TNBC tissues than adjacent normal tissues (Fig. 4C). Next, we upregulated miR-4739 expression through transfection of miR-4739 mimics and inhibited the expression of miR-4739 through transfection of miR-4739 inhibitor (Fig. 4D-E). The binding site between LINC00504 and miR-4739 was predicted from DIANA tool. and mutated the binding site of LINC00504 for miR-479 for the next assay (Fig. 4F). The luciferase activity of wild-type LINC00504 reporters was decreased by miR-6754-5p mimics and increased by miR-4739 inhibitor, while the luciferase activity of LINC00504-Mut reporters showed no significant change (Fig. 4G). Moreover, relative LINC00504 enrichment precipitated by anti-IgG and antiAgo2 was measured using the RIP assay. The finding depicted that miR-4739 mimics elevated the relative enrichment of LINC00504 precipitated by anti-Ago2, indicating that LINC00504 could interact with miR4739 (Fig. 4H). Thus, LINC00504 is a molecular sponge for miR-4739.

\subsection{KLK4 is the downstream target of miR-4739 and activates the Wnt/ $\beta$-catenin pathway}

To detect the potential target of miR-4739, we predicted five mRNAs which might interact with miR-4739 using TargetScan (http://www.targetscan.org/vert_72/) (Fig. 5A). After overexpressing miR-4739 using miR-4739 mimics, KLK4 expression exhibited the most significant downregulation, while expression of other four candidate mRNAs depicted no significant changes (Fig. 5B). The upregulation of KLK4 expression in TNBC cells and tissues was validated by RT-qPCR (Fig. 5C-D). Furthermore, upregulation of miR-4739 repressed the KLK4 expression and upregulation of LINC00504 elevated KLK4 expression; miR4739 inhibition increased KLK4 expression and silencing of LINC00504 reduced KLK4 expression (Fig. 5E). Results of RIP assay revealed that LINC00504, miR-4739 and KLK4 were enriched in anti-Ago2 group in comparison with the control group, revealing that miR-4739 could interact with LINC00504 and KLK4 (Fig. 5F). Subsequently, the binding sequence between miR-4739 and 3'UTR of KLK4 were 
identified, and the binding site was mutated for the luciferase reporter assay (Fig. 5G). Luciferase activity of the KLK4 3'UTR-WT reporter was suppressed by the miR-4739 overexpression using miR-4739 mimics and elevated by the miR-4739 downregulation using miR-4739 inhibitor. However, no significant change in luciferase activity of KLK4 3'UTR-Mut was detected (Fig. 5H). Moreover, the SKBR-3 and MDA-MB-231 cells were transfected by sh-LINC00504\#1/2. Transfection of sh-LINC00504\#1/2 significantly reduced KLK4 expression (Fig. 5I). KLK4 has been documented to activate the Wnt/ $\beta$-catenin pathway [21]. We aimed to investigate whether LINC00505 could activate the Wnt/ $\beta$-catenin pathway via regulating KLK4. It was revealed from TOP/FOP flash assay that silencing of LINC00504 reduced the luciferase activity of TOP vector, while such effect was reversed by pcDNA3.1-KLK4, indicating that LINC00504 activated the Wnt/ $\beta$-catenin pathway by upregulation of KLK4 (Fig. $5 \mathrm{~J}$ ). Importantly, overexpression of KLK4 reversed the suppressive effects of sh-LINC00504\#1/2 on protein levels of $\beta$-catenin and c-Myc, suggesting that LINC00504 activated the Wnt/ $\beta$-catenin pathway through upregulating KLK4 (Fig. 5K). In summary, these results showed that LINC00504 could directly interact with miR-4739 to upregulate KLK4 and trigger the $\mathrm{Wnt} / \beta$-catenin pathway.

\subsection{The LINC00504/miR-4739/KLK4 axis promotes cell growth and cell cycle progression as well as suppresses cell apoptosis in TNBC}

Finally, we decided to explore whether LINC00504 exerts its biological function in TNBC cells via upregulation of KLK4. CCK-8 and colony formation assays confirmed that KLK4 upregulation increased cell viability and proliferation, which were previously decreased by sh-LINC00504\#1; knockdown of miR4739 also counteracted the suppressive effects of sh-LINC00504\#1 on cell viability and proliferation (Fig. 6A-B). Then, the influence of silencing LINC00504 on cell cycle was reversed by overexpression of KLK4 or downregulation of miR-4739 in MDA-MB-231 cells (Fig. 6C). Subsequently, we detected cell apoptosis rate through flow cytometry analysis and apoptosis-related protein levels through western blot analysis in MDA-MB-231 cells. It was demonstrated that the increased cell apoptosis caused by shLINC00504\#1 was further decreased by KLK4 overexpression or miR-4739 downregulation (Fig. 6D-E). Finally, KLK4 overexpression or miR-4739 downregulation countervailed the suppressive effect of shLINC00504\#1 on caspase-3 activity (Fig. 6F). These results confirmed that the LINC00504 could promote cell viability and cell cycle progression and suppress cell apoptosis through the positive modulation on KLK4 at a miR-4739-dependent manner.

\section{Discussion}


This study provides the material significance for LINC00504 in TNBC cells. The results revealed that LINC00504 was upregulated in TNBC tissues and cell lines. LINC00504 knockdown suppressed TNBC cell proliferation, leading to cell cycle arrest in the G0/G1 phase, and accelerated cell apoptosis in TNBC cells. Our study subsequently indicated that STAT3 was the transcription factor for LINC00504. STAT3 serves as the transcription factor for IncRNA SNHG17 [22]. Moreover, STAT3 also plays an important role in TNBC. LEPRb-induced STAT3 activation is critical for the induction and maintenance of TNBC cancer stem cells [23].

The ceRNA pattern is a typical regulatory mechanism in progression of multiple cancers, including TNBC $[19,24]$. Thus, we further investigated the underlying ceRNA network of LINC00504 in TNBC. Our findings showed that LINC00504 acted as a molecular sponge of miR-4739., whose expression is negatively regulated by VPS9D1-AS1 in prostate cancer cells [25]. Moreover, other two investigations have documented the functions of miR-4739, which is associated with unilineage hematopoietic stem cell differentiation, the $\beta$-catenin signaling pathway and gastric cancer, as well as in the pathogenesis of negative anaplastic large cell lymphoma $[26,27]$. Our study revealed that KLK4 served as the direct target of miR-4739. LINC00504 competitively interacted with miR-4739 to upregulate KLK4. Additionally, KLK4 protein expression could play the role as a selective predictive tissue-based biomarker on breast cancer patient's response to adjuvant systemic cancer therapy [28].

The Wnt/ $\beta$-catenin pathway can be activated in plentiful cellular processes, including cell proliferation, differentiation, migration and polarity, survival and self-renewal in stem cells. As a complicated intracellular cell signaling cascade, the Wnt/ $\beta$-catenin pathway is confirmed as a highly conserved and tightly regulated signaling that controls carcinogenesis in various cancers $[29,30]$. It was declared that the Wnt/ $\beta$-catenin pathway participated in TNBC progression. HePTP facilitates migration and invasion ability of TNBC cells via triggering the Wnt/ $\beta$-catenin signaling pathway [31]. LGR6 promotes tumor proliferation and metastasis through the Wnt/ $\beta$-catenin signaling pathway in TNBC [32]. Abundant studies have depicted that KLK4 can activate the Wnt/ $\beta$-catenin signaling. Silencing of KLK4 inhibits the growth of oral squamous cell carcinoma cells through the Wnt/ $\beta$-catenin signaling pathway [33]. Overexpression of LINC01314 down-regulated KLK4 to suppress the Wnt/ $\beta$-catenin signaling pathway in gastric cancer [21]. Our study implied that KLK4 activated the Wnt/ $\beta$-catenin pathway in TNBC cells and that LINC00504 triggered the Wnt/ $\beta$-catenin pathway through upregulation of KLK4. After rescue assays, we demonstrated that KLK4 could counteract the influences of LINC00504 downregulation on cell proliferation, cell cycle progression, cell apoptosis, revealing that LINC00504 promoted TNBC cell malignant behaviors via overexpression of KLK4 to activate the Wnt/ $\beta$-catenin pathway.

In summary, we elucidated that the LINC00504/miR-4739/KLK4 axis promotes TNBC cell proliferation ability, cell cycle and suppresses cell apoptosis through the Wnt/ $\beta$-catenin pathway, which may shed a new insight into TNBC treatment.

\section{Abbreviations}


IncRNAs

long non-coding RNAs

TNBC

triple-negative breast cancer

ceRNAs

competing endogenous RNAs

miRNAs

microRNAs

GAPDH

glyceraldehyde phosphate dehydrogenase

RT-qPCR

Reverse transcription quantitative polymerase chain reaction

CCK-8

cell counting kit-8

$\mathrm{PI}$

propidium iodide

RIPA

RNA immunoprecipitation assay

SDS-PAGE

sodium dodecyl sulfate, polyacrylamide gel electrophoresis

ChIP

Chromatin immunoprecipitation

RIP

RNA immunoprecipitation

$\mathrm{NC}$

negative control

SD

standard deviation

\section{Declarations}

Ethics approval and consent to participate

This research was approved by the Ethics Committee of the First Hospital of Jilin University (Jilin, China).

Consent to publish

Not applicable.

\section{Availability of data and materials}


The datasets applied during the present study is available from the corresponding author on reasonable request.

\section{Competing interests}

The authors declare that no conflict of interest exists.

\section{Funding}

This research did not receive any specific grant from funding agencies in the public, commercial, or nonprofit sectors.

\section{Authors' contributions}

Study concepts and design: D.W. and H.J.;

Data acquisition and analysis: Z.Z. and S.L.;

Experimental studies and statistical analysis: D.W. and H.J.;

Manuscript preparation and editing: D.W. and H.J.;

Manuscript review: D.W., H.J., Z.Z. and S.L.

All authors approved final version of manuscript.

\section{Acknowledgements}

We thank all participators for their help.

\section{Authors' information}

Di Wu, E-mail: wu_di@jlu.edu.cn

Hongyao Jia, E-mail: jhy@jlu.edu.cn

Zhiru Zhang, E-mail: zaeeoql@163.com

Sijie Li, E-mail: lisij@jlu.edu.cn

\section{References}

1. Kischel P, Girault A, Rodat-Despoix L, Chamlali M, Radoslavova S, Abou Daya H, Lefebvre T, Foulon A, Rybarczyk P, Hague F, et al: Ion Channels: New Actors Playing in Chemotherapeutic Resistance. Cancers 2019, 11(3). 
2. Zhang Y, Zhang S, Yin J, Xu R. MiR-566 mediates cell migration and invasion in colon cancer cells by direct targeting of PSKH1. Cancer Cell Int. 2019;19:333.

3. Lin NU, Vanderplas A, Hughes ME, Theriault RL, Edge SB, Wong YN, Blayney DW, Niland JC, Winer EP, Weeks JC. Clinicopathologic features, patterns of recurrence, and survival among women with triplenegative breast cancer in the National Comprehensive Cancer Network. Cancer. 2012;118(22):546372.

4. Bianchini G, Balko JM, Mayer IA, Sanders ME, Gianni L. Triple-negative breast cancer: challenges and opportunities of a heterogeneous disease. Nat Rev Clin Oncol. 2016;13(11):674-90.

5. Lyons TG. Targeted Therapies for Triple-Negative Breast Cancer. Curr Treat Options Oncol. 2019;20(11):82.

6. Hamilton MJ, Young M, Jang K, Sauer S, Neang VE, King AT, Girke T, Martinez E. HOTAIRM1 IncRNA is downregulated in clear cell renal cell carcinoma and inhibits the hypoxia pathway. Cancer letters. 2020;472:50-8.

7. Wu D, Zhang T, Wang J, Zhou J, Pan H, Qu P. Long noncoding RNA NNT-AS1 enhances the malignant phenotype of bladder cancer by acting as a competing endogenous RNA on microRNA-496 thereby increasing HMGB1 expression. Aging. 2019;11(24):12624-40.

8. Luo K, Geng J, Zhang Q, Xu Y, Zhou X, Huang Z, Shi KQ, Pan C, Wu J. LncRNA CASC9 interacts with CPSF3 to regulate TGF- $\beta$ signaling in colorectal cancer. Journal of experimental clinical cancer research: CR. 2019;38(1):249.

9. Zhou Z, Lin Z, He Y, Pang X, Wang Y, Ponnusamy M, Ao X, Shan P, Tariq MA, Li P, et al: The Long Noncoding RNA D63785 Regulates Chemotherapy Sensitivity in Human Gastric Cancer by Targeting miR-422a. Molecular therapy Nucleic acids 2018, 12:405-419.

10. Li J, Li Q, Li D, Shen Z, Zhang K, Bi Z, Li Y: Long Non-Coding RNA MNX1-AS1 Promotes Progression of Triple Negative Breast Cancer by Enhancing Phosphorylation of Stat3. Frontiers in oncology 2020, 10:1108.

11. Dong F, Ruan S, Wang J, Xia Y, Le K, Xiao X, Hu T, Wang Q. M2 macrophage-induced IncRNA PCAT6 facilitates tumorigenesis and angiogenesis of triple-negative breast cancer through modulation of VEGFR2. Cell death disease. 2020;11(9):728.

12. Zhang $M$, Wang $N$, Song $P$, Fu Y, Ren $Y$, Li Z, Wang J. LncRNA GATA3-AS1 facilitates tumour progression and immune escape in triple-negative breast cancer through destabilization of GATA3 but stabilization of PD-L1. Cell proliferation 2020:e12855.

13. Ma HP, Wang LX, Li W, Guo HH, Wu Y, Li XY. Upregulation of LINC00504 is associated with aggressive progression and poor prognosis in non-small cell lung cancer. Eur Rev Med Pharmacol Sci. 2020;24(2):699-703.

14. Liu Y, He X, Chen Y, Cao D. Long non-coding RNA LINC00504 regulates the Warburg effect in ovarian cancer through inhibition of miR-1244. Molecular cellular biochemistry. 2020;464:39-50.

15. Feng J, Ma J, Liu S, Wang J, Chen Y. A noncoding RNA LINC00504 interacts with c-Myc to regulate tumor metabolism in colon cancer. Journal of cellular biochemistry. 2019;120(9):14725-34. 
16. Zhang Y, Yu F, Bao S, Sun J. Systematic Characterization of Circular RNA-Associated CeRNA Network Identified Novel circRNA Biomarkers in Alzheimer's Disease. Front Bioeng Biotechnol. 2019;7:222.

17. Dobosz P, Stempor PA, Roszik J, Herman A, Layani A, Berger R, Avni D, Sidi Y, Leibowitz-Amit R. Checkpoint Genes at the Cancer Side of the Immunological Synapse in Bladder Cancer. Translational oncology. 2020;13(2):193-200.

18. Li S, Wu D, Jia H, Zhang Z. Long non-coding RNA LRRC75A-AS1 facilitates triple negative breast cancer cell proliferation and invasion via functioning as a ceRNA to modulate BAALC. Cell death disease. 2020;11(8):643.

19. Hua K, Deng X, Hu J, Ji C, Yu Y, Li J, Wang X, Fang L. Long noncoding RNA HOST2, working as a competitive endogenous RNA, promotes STAT3-mediated cell proliferation and migration via decoying of let-7b in triple-negative breast cancer. Journal of experimental clinical cancer research: CR. 2020;39(1):58.

20. Yang F, Shen Y, Zhang W, Jin J, Huang D, Fang H, Ji W, Shi Y, Tang L, Chen W, et al. An androgen receptor negatively induced long non-coding RNA ARNILA binding to miR-204 promotes the invasion and metastasis of triple-negative breast cancer. Cell death differentiation. 2018;25(12):2209-20.

21. Tang L, Wen JB, Wen P, Li X, Gong M, Li Q. Long non-coding RNA LINC01314 represses cell migration, invasion, and angiogenesis in gastric cancer via the Wnt/ $\beta$-catenin signaling pathway by downregulating KLK4. Cancer cell international. 2019;19:94.

22. Pan X, Guo Z, Chen Y, Zheng S, Peng M, Yang Y, Wang Z. STAT3-Induced IncRNA SNHG17 Exerts Oncogenic Effects on Ovarian Cancer through Regulating CDK6. Molecular therapy Nucleic acids. 2020;22:38-49.

23. Thiagarajan $P$, Zheng Q, Bhagrath $M$, Mulkearns-Hubert E, Myers M, Lathia J, Reizes O. STAT3 activation by leptin receptor is essential for TNBC stem cell maintenance. Endocrine-related Cancer. 2017;24(8):415-26.

24. Zhou Q, Guo J, Huang W, Yu X, Xu C, Long X: Linc-ROR promotes the progression of breast cancer and decreases the sensitivity to rapamycin through miR-194-3p targeting MECP2. Molecular oncology 2020, 14(9):2231-2250.

25. Wang X, Chen Q, Wang X, Li W, Yu G, Zhu Z, Zhang W: ZEB1 activated-VPS9D1-AS1 promotes the tumorigenesis and progression of prostate cancer by sponging miR-4739 to upregulate MEF2D. Biomedicine \& pharmacotherapy = Biomedecine \& pharmacotherapie 2020, 122:109557.

26. Cattaneo M, Pelosi E, Castelli G, Cerio A, D'Angiò A, Porretti L, Rebulla P, Pavesi L, Russo G, Giordano A, et al. A miRNA Signature in Human Cord Blood Stem and Progenitor Cells as Potential Biomarker of Specific Acute Myeloid Leukemia Subtypes. Journal of cellular physiology. 2015;230(8):1770-80.

27. Wang C, Chen X, Chen X, He Y, Cao L. [Expression of microRNA in ALK-negative anaplastic large cell lymphoma and CD30-positive peripheral T cell lymphoma, not otherwise specified]. Zhonghua Bing Li Xue Za Zhi. 2015;44(8):565-70.

28. Yang F, Aubele M, Walch A, Gross E, Napieralski R, Zhao S, Ahmed N, Kiechle M, Reuning U, Dorn J, et al. Tissue kallikrein-related peptidase 4 (KLK4), a novel biomarker in triple-negative breast cancer. 
Biological chemistry. 2017;398(10):1151-64.

29. Rudnicki MA, Williams BO. Wnt signaling in bone and muscle. Bone. 2015;80:60-6.

30. Kahn M. Can we safely target the WNT pathway? Nat Rev Drug Discov. 2014;13(7):513-32.

31. Yu L, Wang C, Pan F, Liu Y, Ren X, Zeng H, Shi Y. HePTP promotes migration and invasion in triplenegative breast cancer cells via activation of $W n t / \beta$-catenin signaling. Biomedicine pharmacotherapy = Biomedecine pharmacotherapie. 2019;118:109361.

32. Kong Y, Ou X, Li X, Zeng Y, Gao G, Lyu N, Liu P. LGR6 Promotes Tumor Proliferation and Metastasis through Wnt/ $\beta$-Catenin Signaling in Triple-Negative Breast Cancer. Molecular therapy oncolytics. 2020;18:351-9.

33. Cui Z, Cui Y, Yang S, Luo G, Wang Y, Lou Y, Sun X. KLK4 silencing inhibits the growth of oral squamous cell carcinoma through Wnt/ $\beta$-catenin signaling pathway. Cell Biol Int. 2017;41(4):392404.

\section{Figures}


A

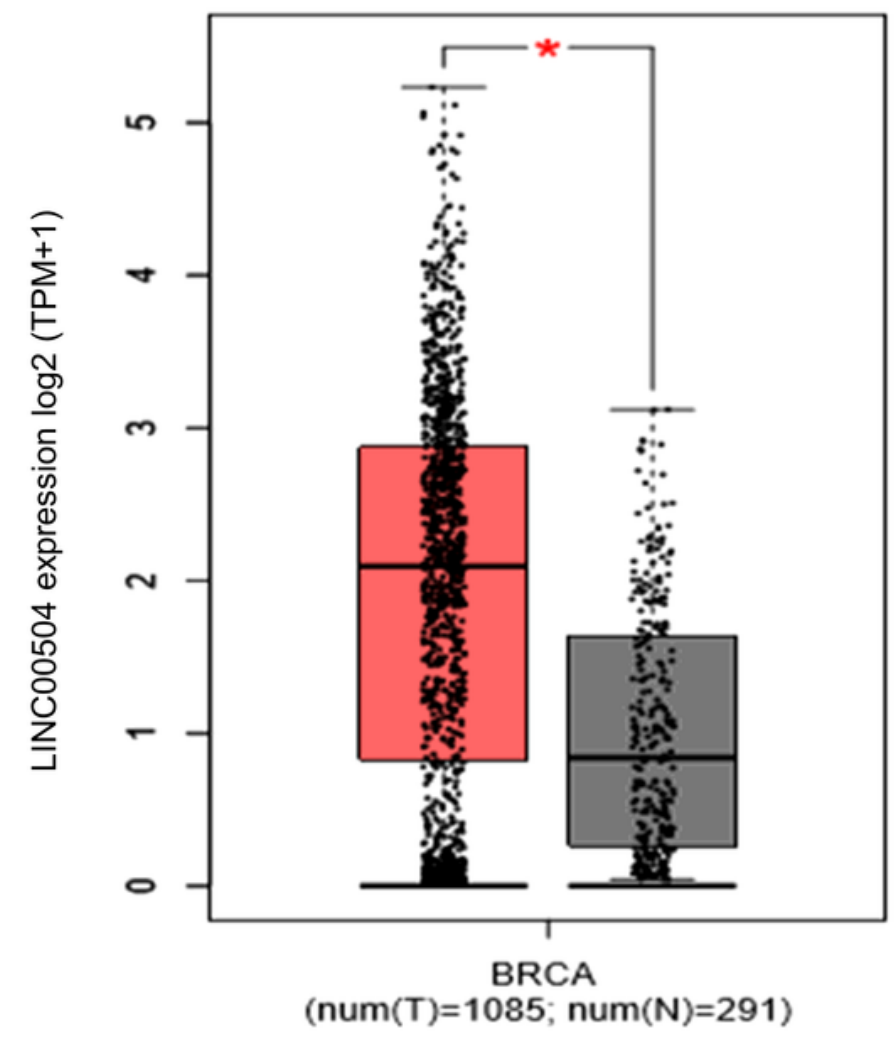

C

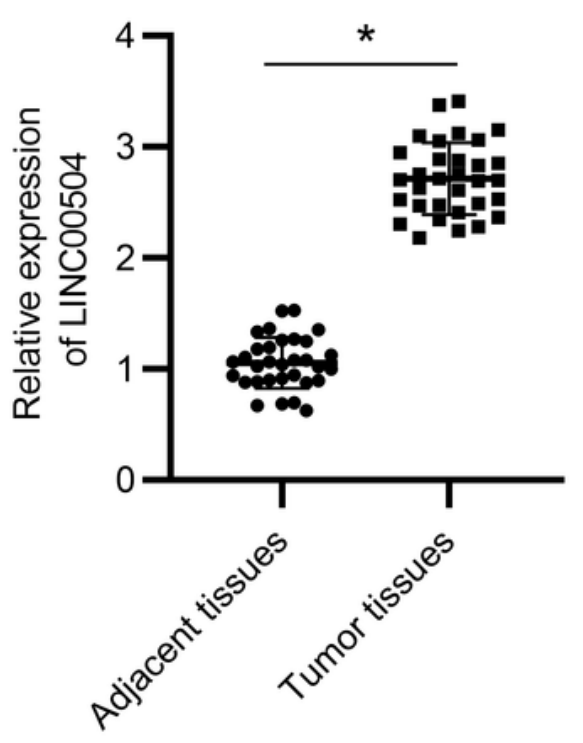

B
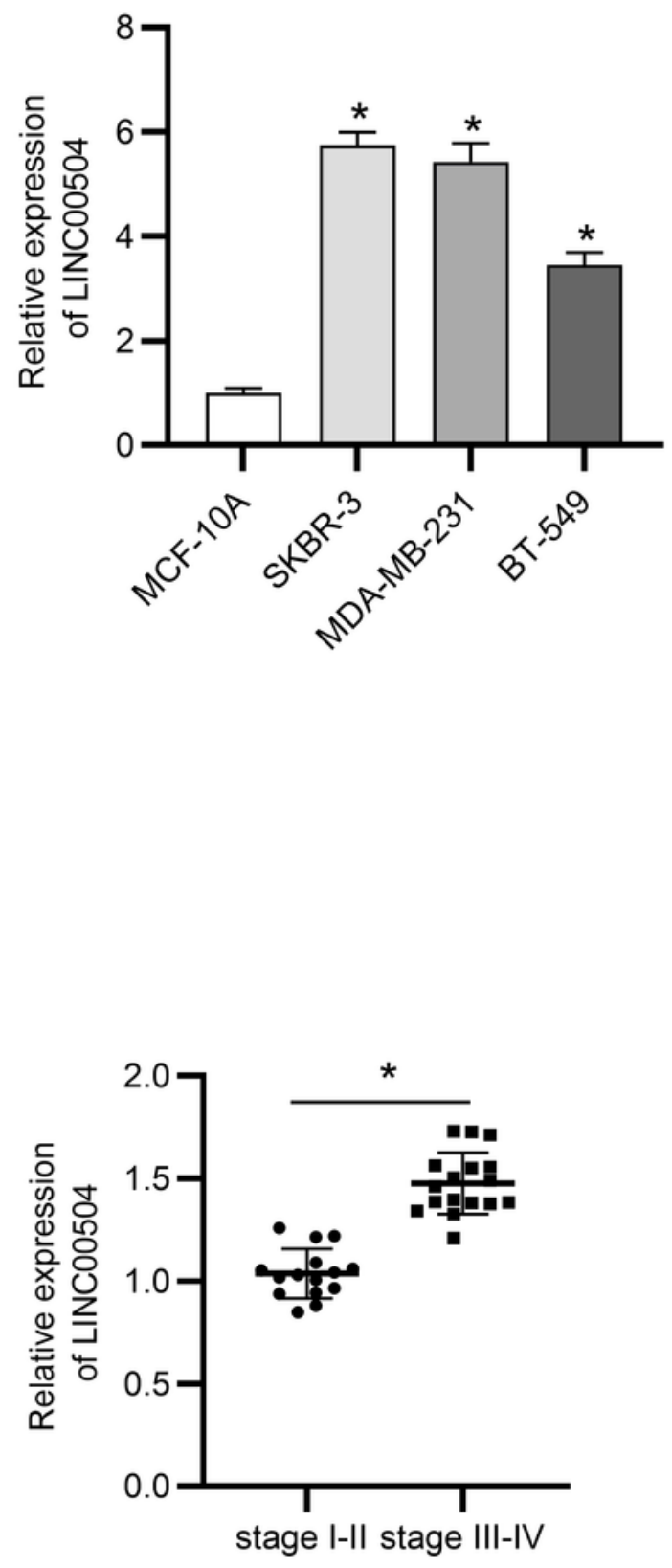

Figure 1

LncRNA LINC00504 is upregulated in TNBC tissues and cells. (A) The GEPIA database showed the expression of LINC00504 in breast cancer (BRAC). (B) Relative expression of LINC00504 in TNBC cell lines (SKBR-3, MDA-MB-231 and BT-549) and normal breast cell line (MCF-10A). (C) Relative LINC00504 expression in TNBC tissues and adjacent non-tumor breast tissues. $n=32$. (D) Relative expression level of 
LINC00504 in TNBC tissues with stage I-II was examined by RT-qPCR $(n=15)$ or stage III-IV $(n=17) .{ }^{*} \mathrm{p} \rrbracket$ 0.05 .

A

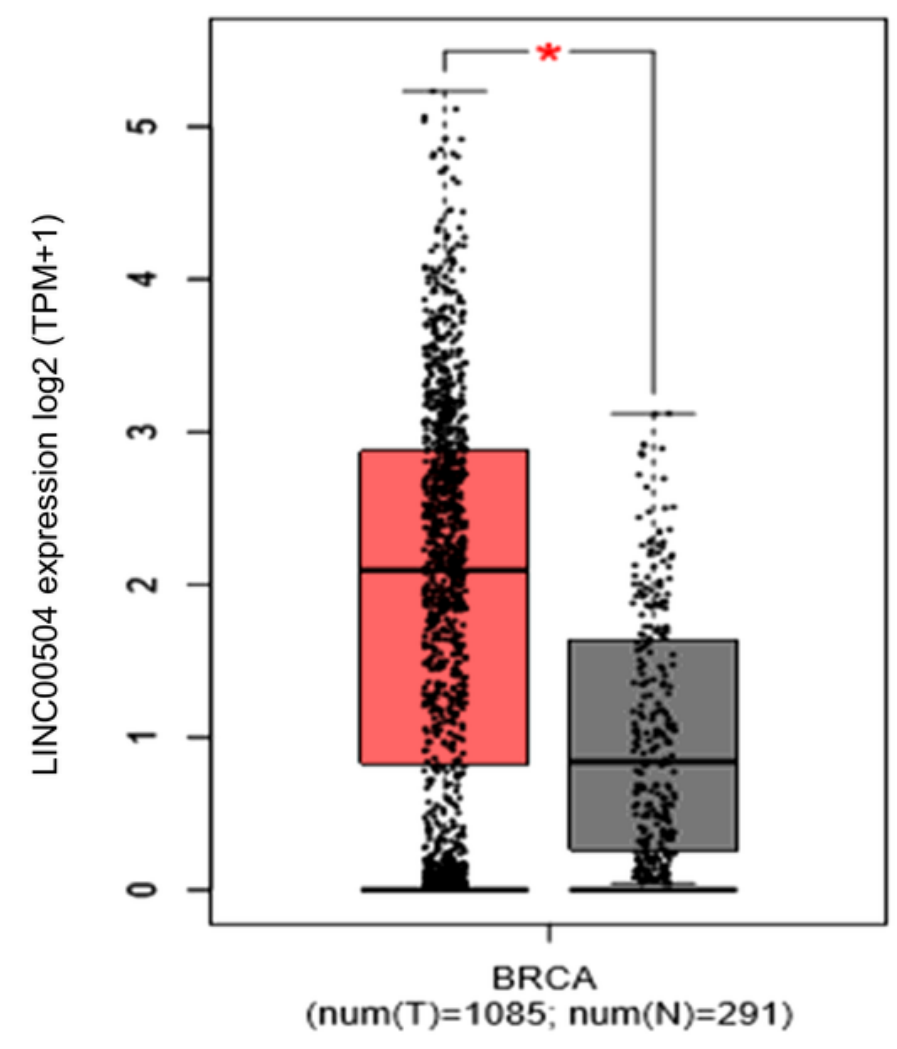

C

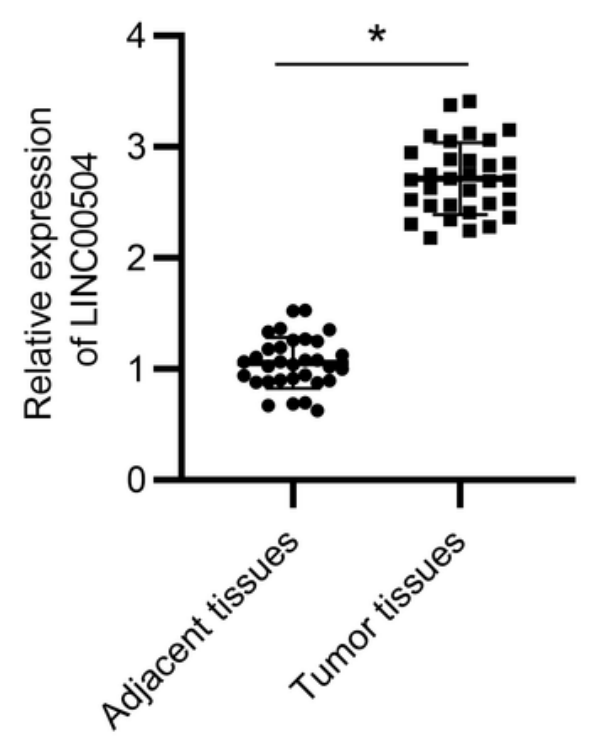

B
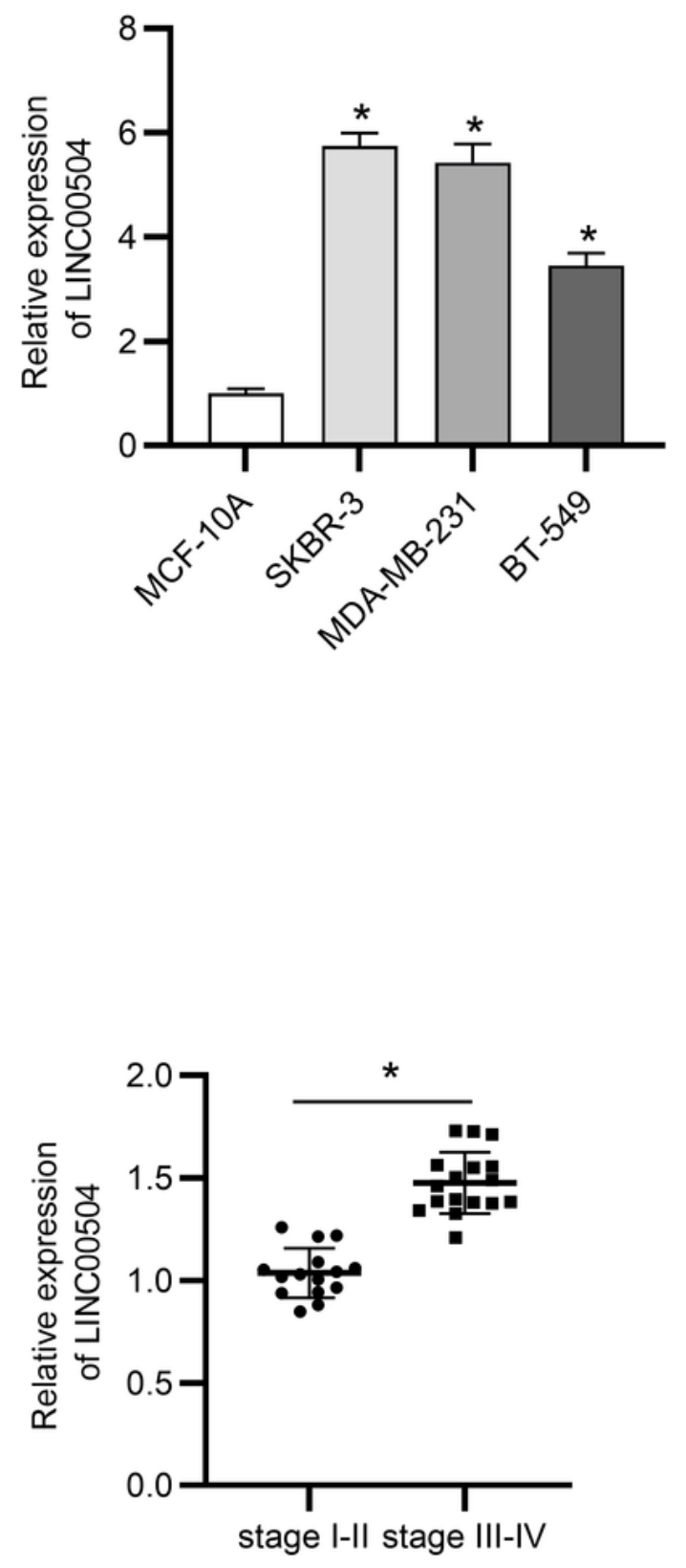

Figure 1

LncRNA LINC00504 is upregulated in TNBC tissues and cells. (A) The GEPIA database showed the expression of LINC00504 in breast cancer (BRAC). (B) Relative expression of LINC00504 in TNBC cell lines (SKBR-3, MDA-MB-231 and BT-549) and normal breast cell line (MCF-10A). (C) Relative LINC00504 
expression in TNBC tissues and adjacent non-tumor breast tissues. $n=32$. (D) Relative expression level of LINC00504 in TNBC tissues with stage I-II was examined by RT-qPCR $(n=15)$ or stage III-IV $(n=17)$. *p》 0.05 .
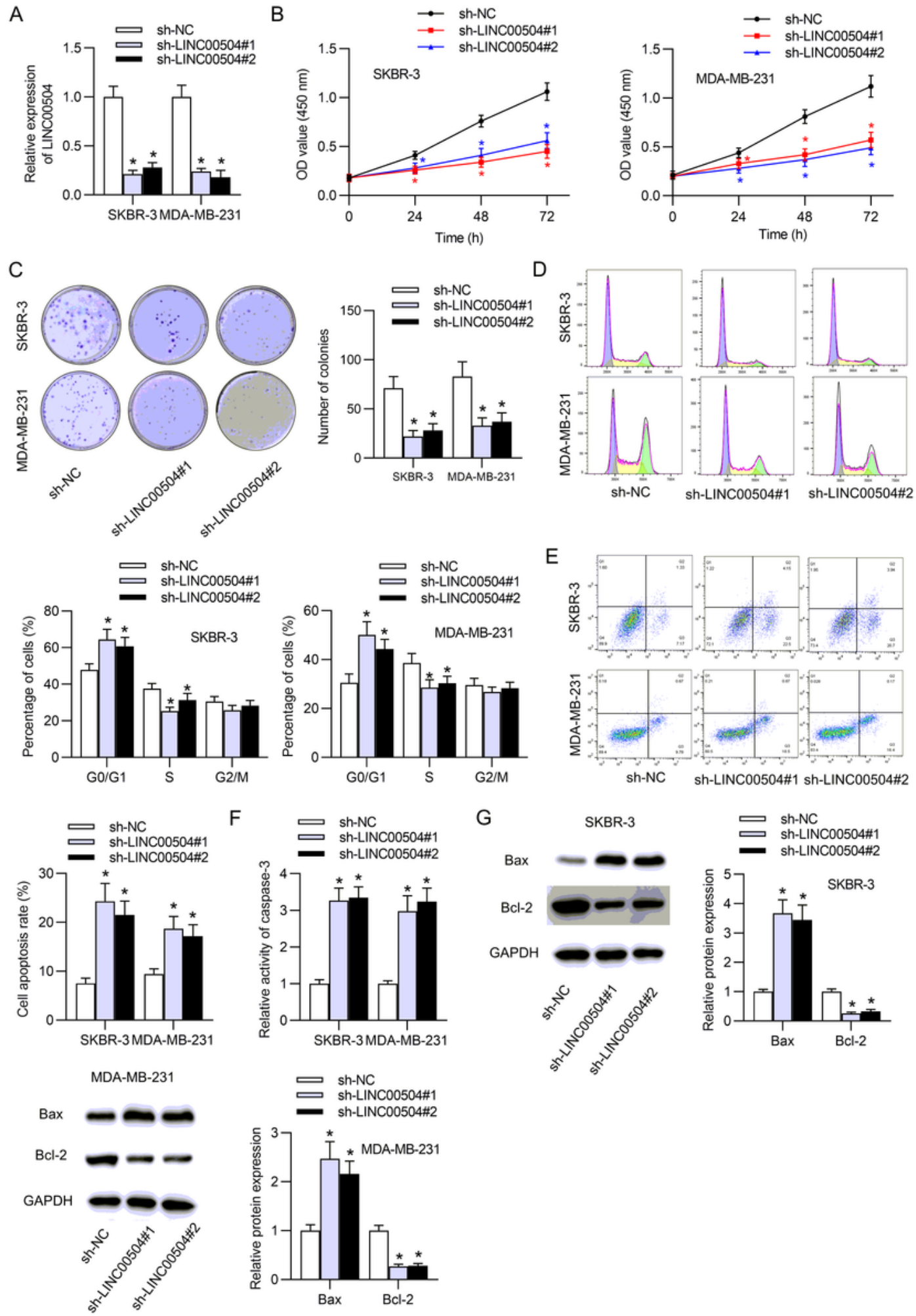

Figure 2

Downregulation of LINC00504 suppresses TNBC cell proliferation and triggers TNBC cell cycle arrest and cell apoptosis. (A) RT-qPCR determined relative expression of LINC00504 in MDA-MB-231 cells and SKBR- 
3 cells after silencing LINC00504. (B) CCK-8 assays measured cell viability of SKBR-3 and MDA-MB-231 cells after LINC00504 knockdown. (C) Colony formation assays measured the colony numbers of SKBR-3 and MDA-MB-231 cells. (D) Flow cytometry detected the effect of silencing LINC00504 on cell cycle progression. We revealed percentage of cell population at different phases of cell cycle. (E) Flow cytometry measured cell apoptosis rate. (F) Caspase-3 Colorimetric Assay Kit determined the activity of caspase-3. (G) Levels of Bax and Bcl-2 was examined by western blot. Full-length blots are presented in Supplementary material. The ImageJ software was used to detect densitometry analysis of the band intensity. ${ }^{*} \mathrm{p} \otimes 0.05$. 
A
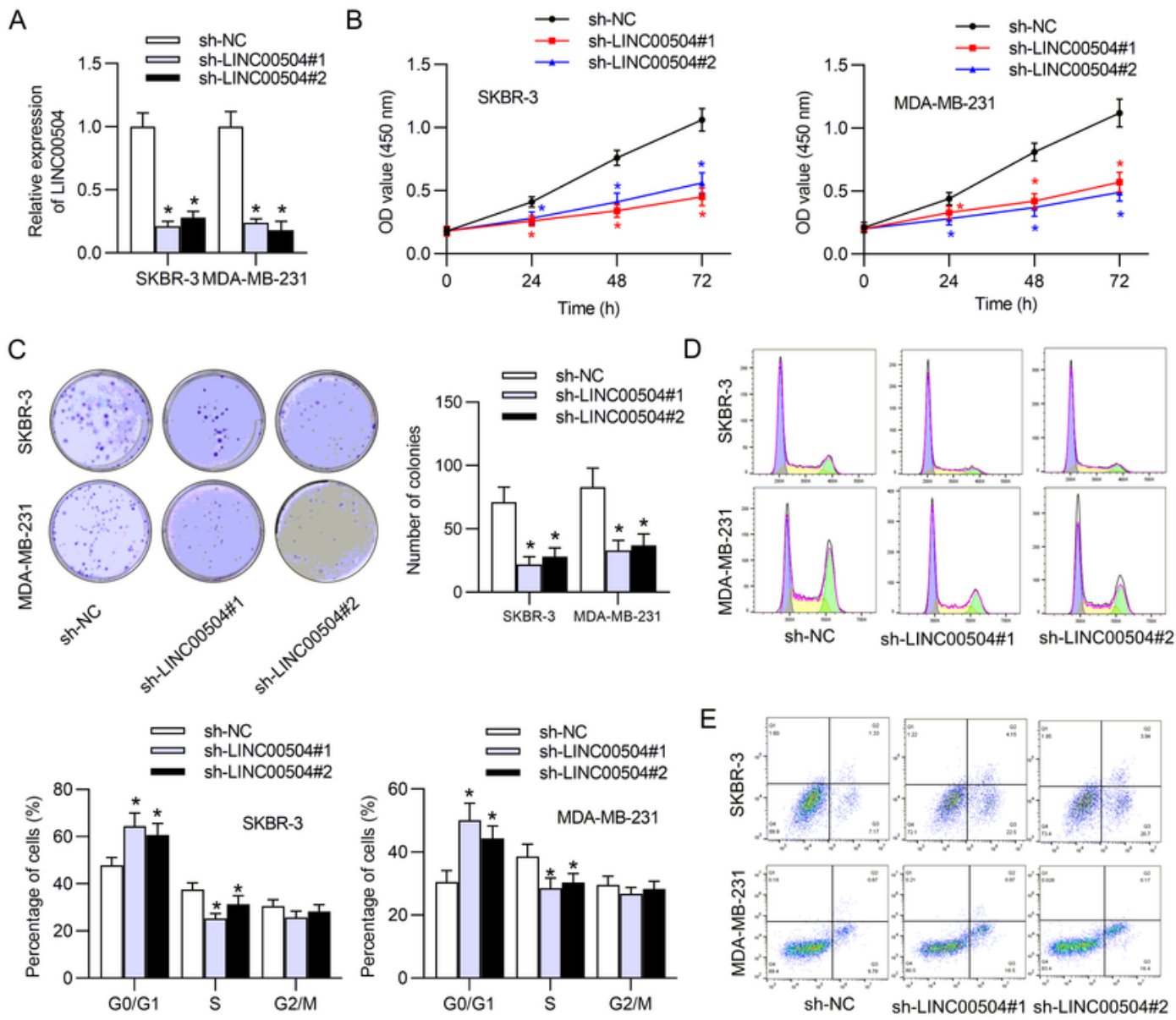

E
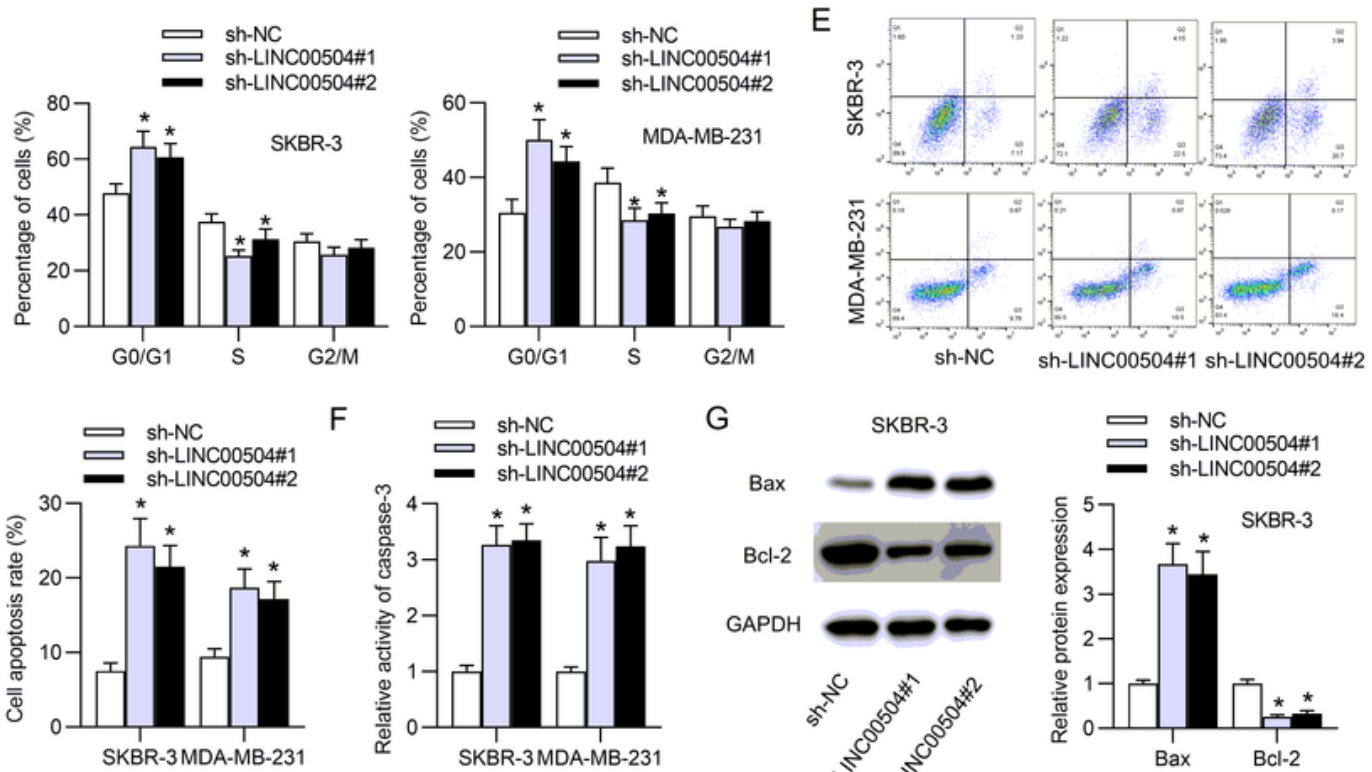

G
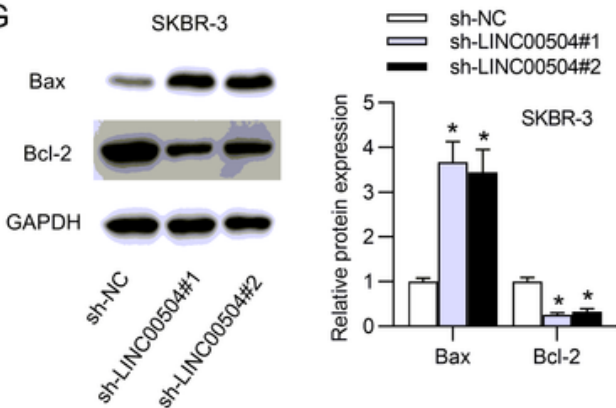
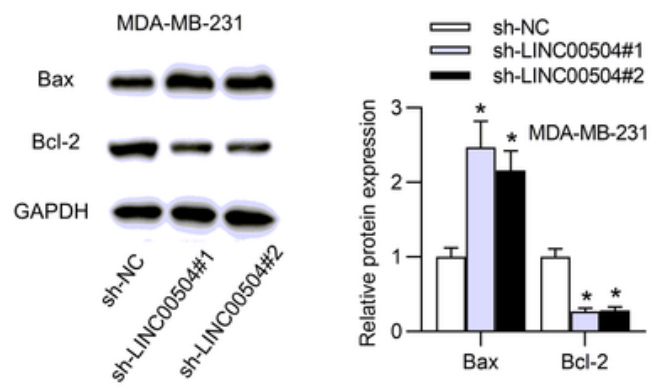

Figure 2

Downregulation of LINC00504 suppresses TNBC cell proliferation and triggers TNBC cell cycle arrest and cell apoptosis. (A) RT-qPCR determined relative expression of LINC00504 in MDA-MB-231 cells and SKBR3 cells after silencing LINC00504. (B) CCK-8 assays measured cell viability of SKBR-3 and MDA-MB-231 cells after LINC00504 knockdown. (C) Colony formation assays measured the colony numbers of SKBR-3 and MDA-MB-231 cells. (D) Flow cytometry detected the effect of silencing LINC00504 on cell cycle 
progression. We revealed percentage of cell population at different phases of cell cycle. (E) Flow cytometry measured cell apoptosis rate. (F) Caspase-3 Colorimetric Assay Kit determined the activity of caspase-3. (G) Levels of Bax and $\mathrm{Bcl}-2$ was examined by western blot. Full-length blots are presented in Supplementary material. The ImageJ software was used to detect densitometry analysis of the band intensity. ${ }^{*} \mathrm{p} \otimes 0.05$.

A

pcDNA3.1
pcDNA3.1-STAT3

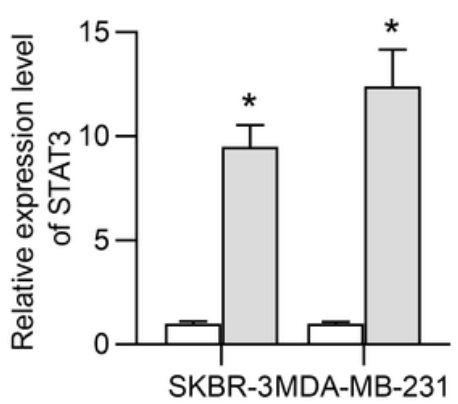

pcDNA3.1

ए pcDNA3.1-STAT3

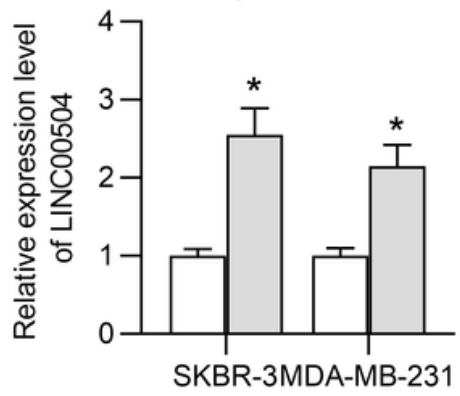

B

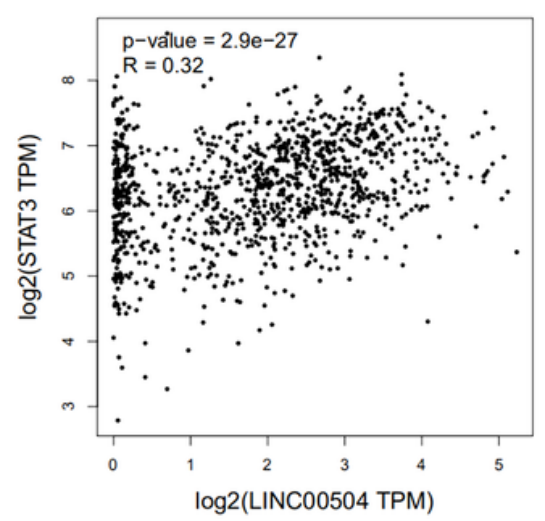

E
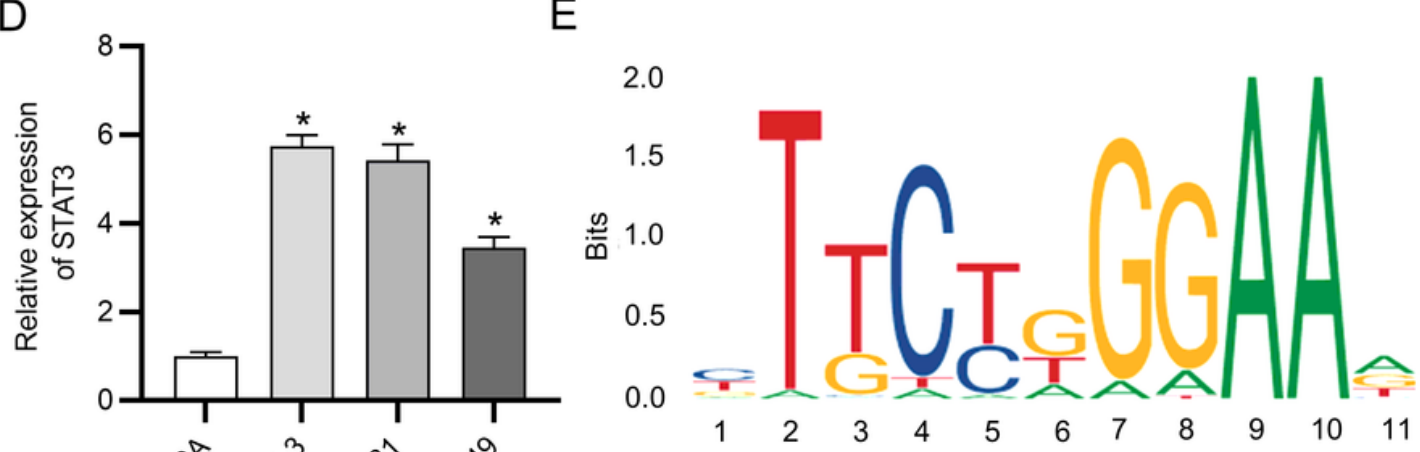

$\mathrm{F}$

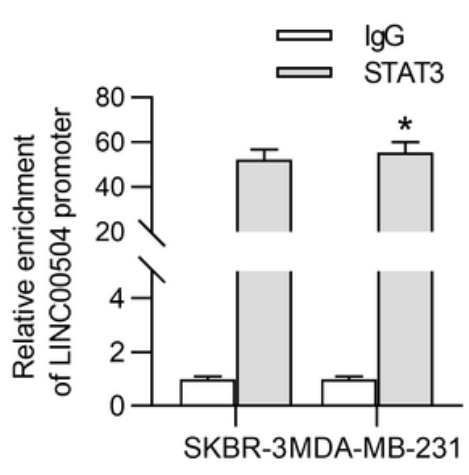

G $\square$ pCDNA3.1

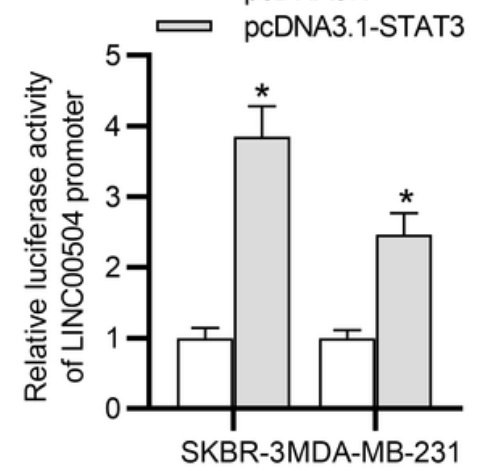

Figure 3

LINC00504 is transcriptionally activated by STAT3. (A) The left panel: overexpression efficiency of STAT3 was verified by RT-qPCR analysis; the right panel: the effects of STAT3 overexpression on LINC00504 expression. (B) The GEPIA database showed the correlation between LINC00504 and STAT3 in breast cancer. (C) RT-qPCR analysis was used to detect the expression of STAT3 in TNBC tissues and adjacent 
normal tissues. (D) The expression of STAT3 in TNBC cells (SKBR-3, MDA-MB-231, BT-549) and normal breast cell line (MCF-10A) was measured through RT-qPCR. (E) DNA motif of STAT3 from JASPAR website. (F) ChIP assay revealed relative expression of LINC00504 promoter pulled down by anti-lgG and anti-STAT3. (G) The luciferase activity of LINC00504 promoter by STAT3 overexpression was manifested

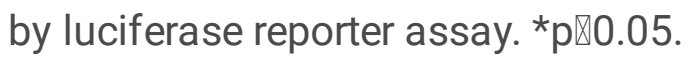

A
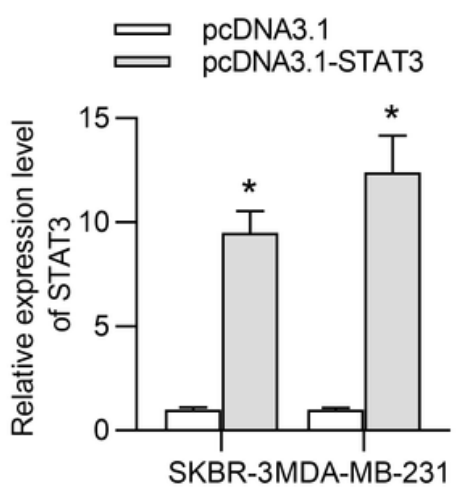

PCDNA3.1 ए pcDNA3.1-STAT3

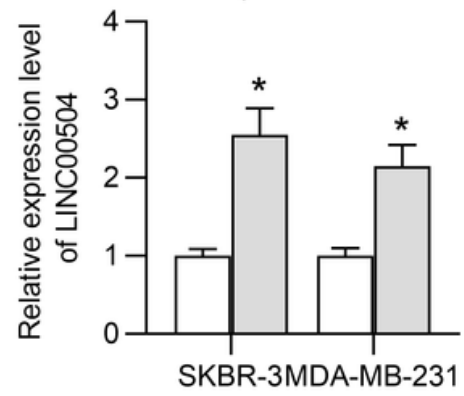

B

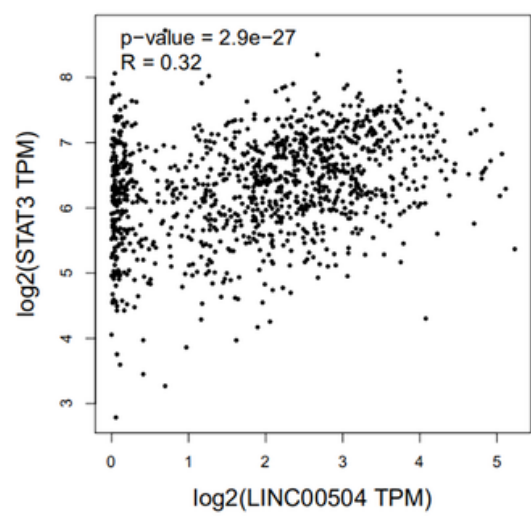

$\mathrm{E}$
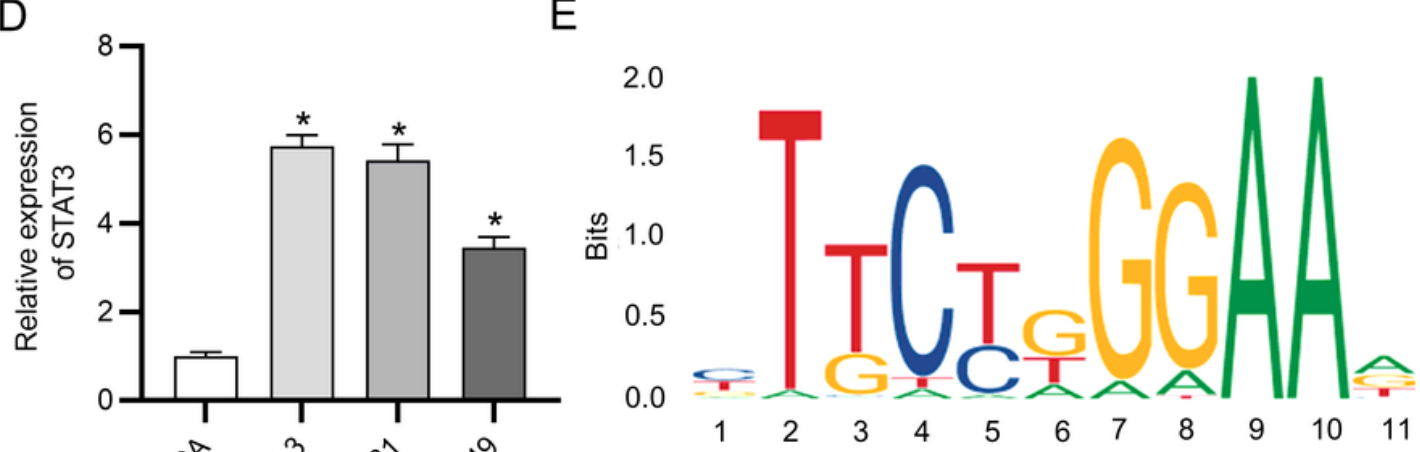

$\mathrm{F}$
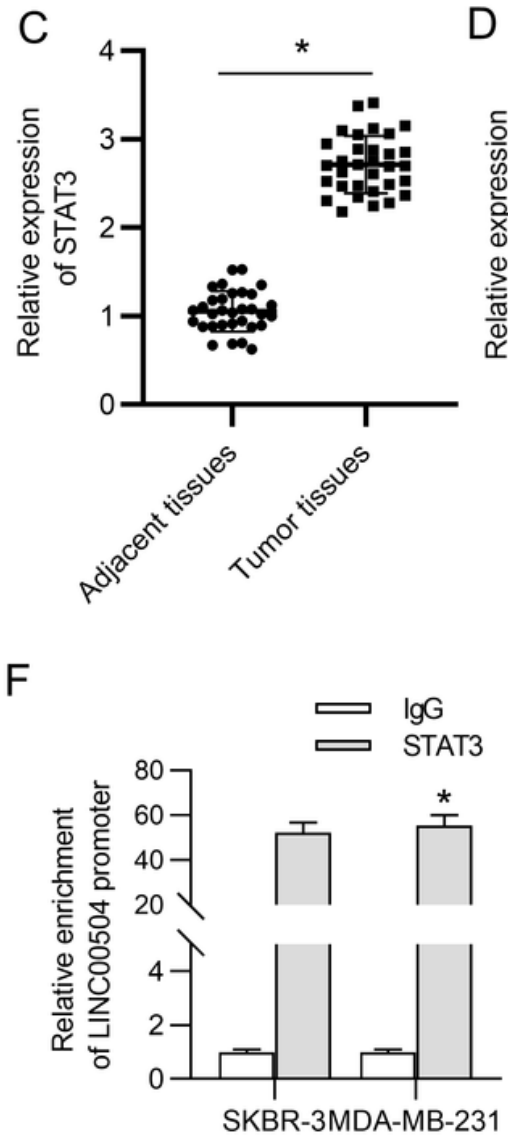

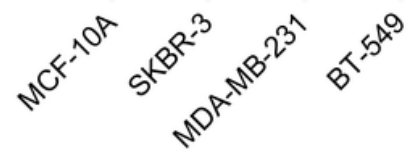

G 2 pcDNA3.1

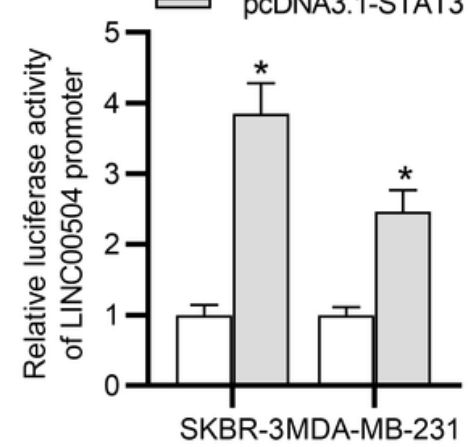

Figure 3

LINC00504 is transcriptionally activated by STAT3. (A) The left panel: overexpression efficiency of STAT3 was verified by RT-qPCR analysis; the right panel: the effects of STAT3 overexpression on LINC00504 expression. (B) The GEPIA database showed the correlation between LINC00504 and STAT3 in breast cancer. (C) RT-qPCR analysis was used to detect the expression of STAT3 in TNBC tissues and adjacent 
normal tissues. (D) The expression of STAT3 in TNBC cells (SKBR-3, MDA-MB-231, BT-549) and normal breast cell line (MCF-10A) was measured through RT-qPCR. (E) DNA motif of STAT3 from JASPAR website. (F) ChIP assay revealed relative expression of LINC00504 promoter pulled down by anti-lgG and anti-STAT3. (G) The luciferase activity of LINC00504 promoter by STAT3 overexpression was manifested by luciferase reporter assay. ${ }^{*} \mathrm{p} \otimes 0.05$.

A ש $\begin{aligned} & \text { nucleus } \\ & \text { cytoplasm }\end{aligned}$

B
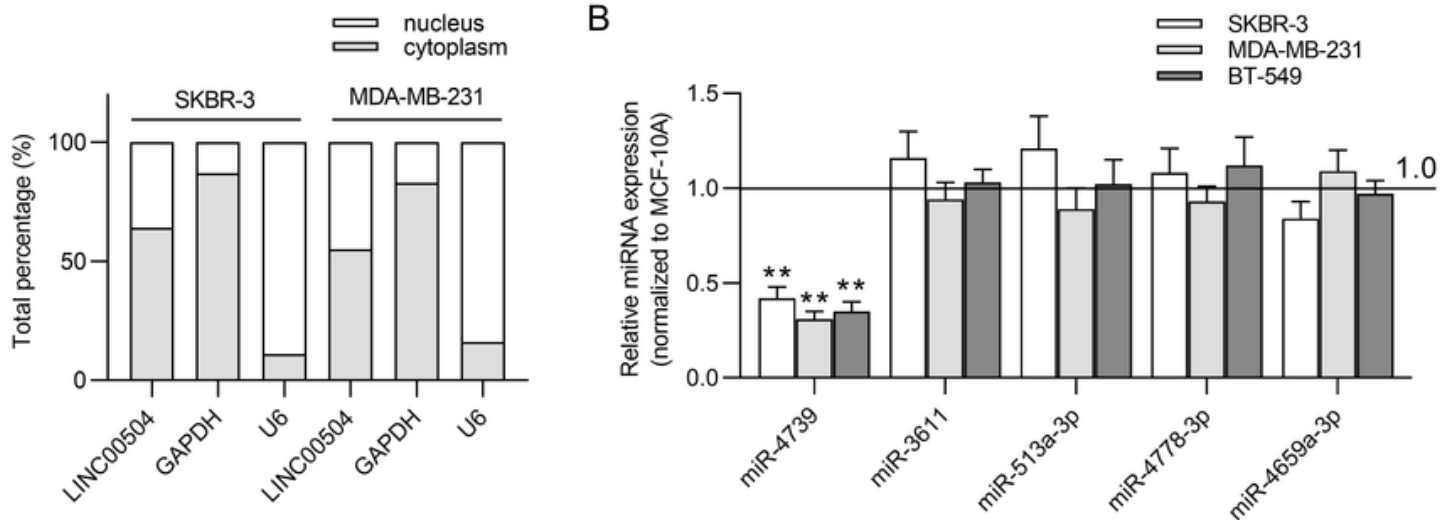

C

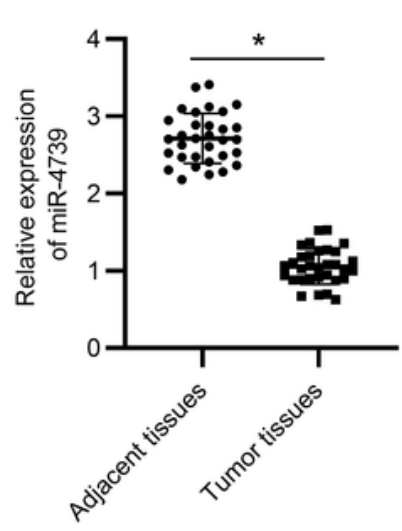

D

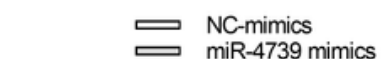

$\mathrm{E}$

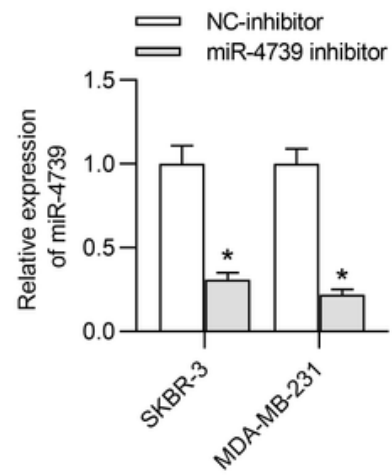

$\mathrm{F}$

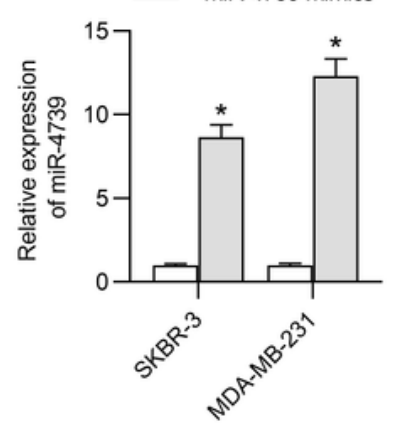

G

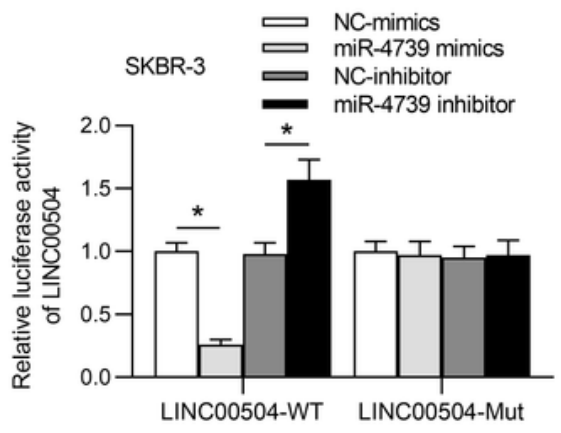

LINC00504-Wt: UUGCUACGAUCA AUUCUGUG UCCUCCCUU $\operatorname{miR}-4739$ : CGGGGAGGCGAGGAGGAGGGA

LINC00504-Mut: UUGCUACGAUUAACGAGACG AGUCGAACG

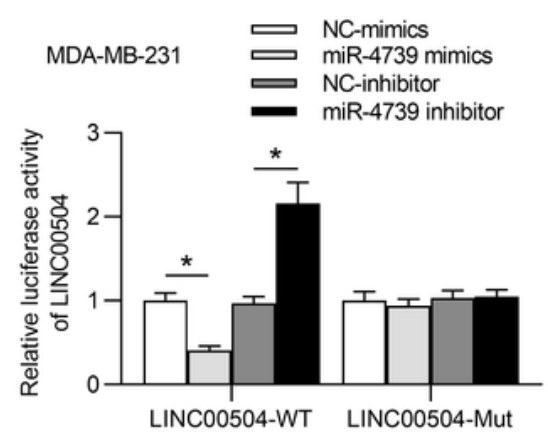

$\mathrm{H}$
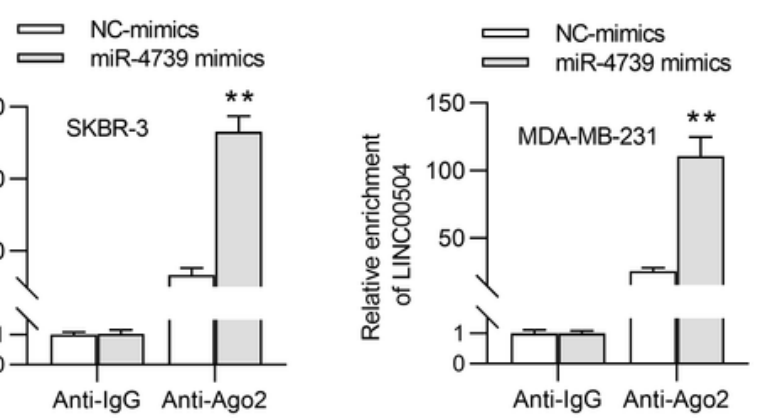

Figure 4 
LINC00504 serves as a molecular sponge of miR-4739. (A) Subcellular fraction assay disclosed the location and distribution of LINC00504 in the cytoplasm and nucleus in TNBC cells. (B) RT-qPCR detected the relative expression of 5 potential miRNAs in TNBC cells. (C) The miR-4739 expression in TNBC tissues and adjacent normal tissues was detected. (D) RT-qPCR measured the relative expression of miR-4739 in NC-mimics, miR-4739 mimics group. (E) RT-qPCR determined the relative expression of miR-4739 in NCinhibitor or miR-4739 inhibitor group. (F) The binding site between miR-4739 and LINC00504 was predicted through starBase v2.0. (G) Luciferase report assay revealed luciferase activity of LINC00504 by miR-4739 mimics or miR-4739 inhibitor. (H) RIP assay revealed relative enrichment of LINC00504 precipitated by anti-IgG and anti-Ago2 in NC-mimics and miR-4739 mimics group. ${ }^{*} \mathrm{p} \otimes 0.05,{ }^{* \star} \mathrm{p}<0.01$. 
A

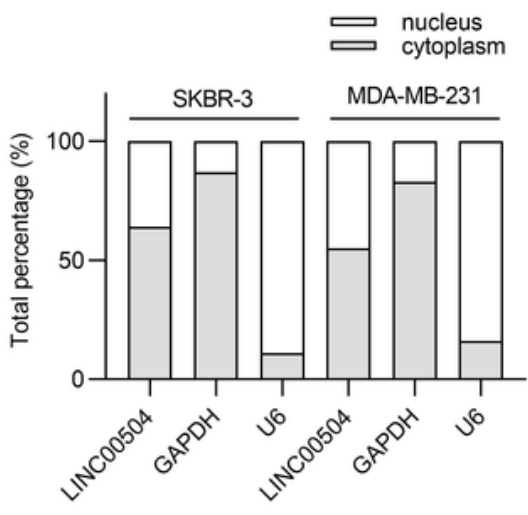

B

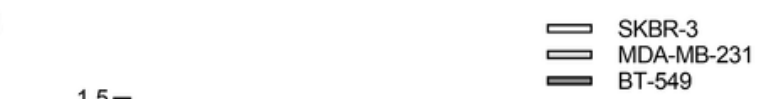

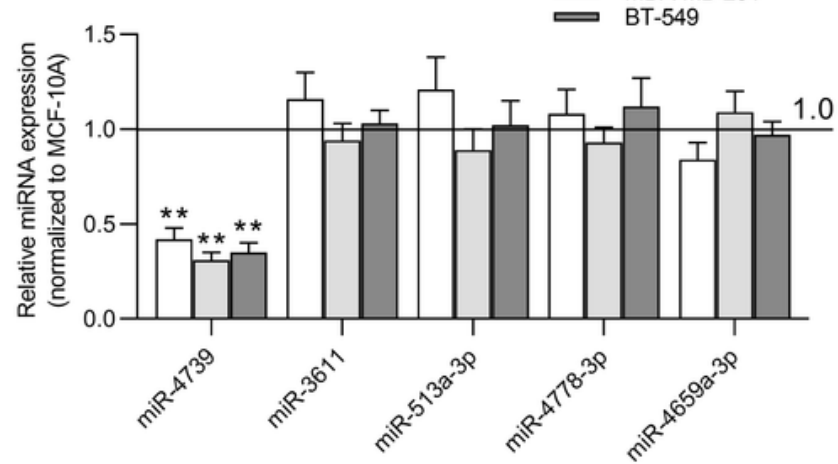

C

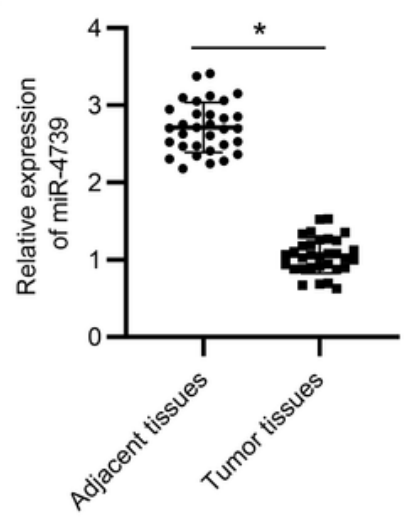

F

LINC00504-Wt: UUgCUACGAUCA AUUCUGUG UCCUCCCUU miR-4739: CGGGGAGGCGAGGAGAGGGAA

LINC00504-Mut: UUGCUACGAUUAACGAGACG AGUCGAACG
D

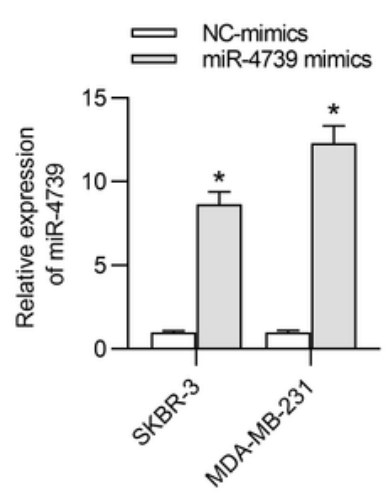

E
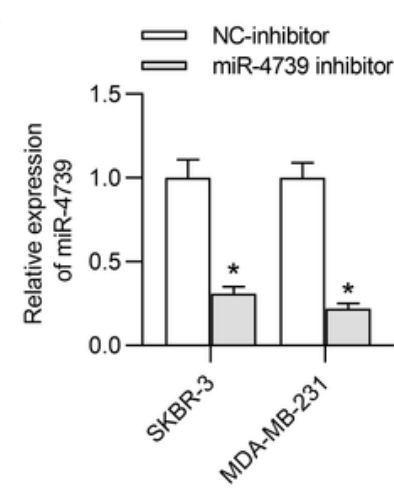

G
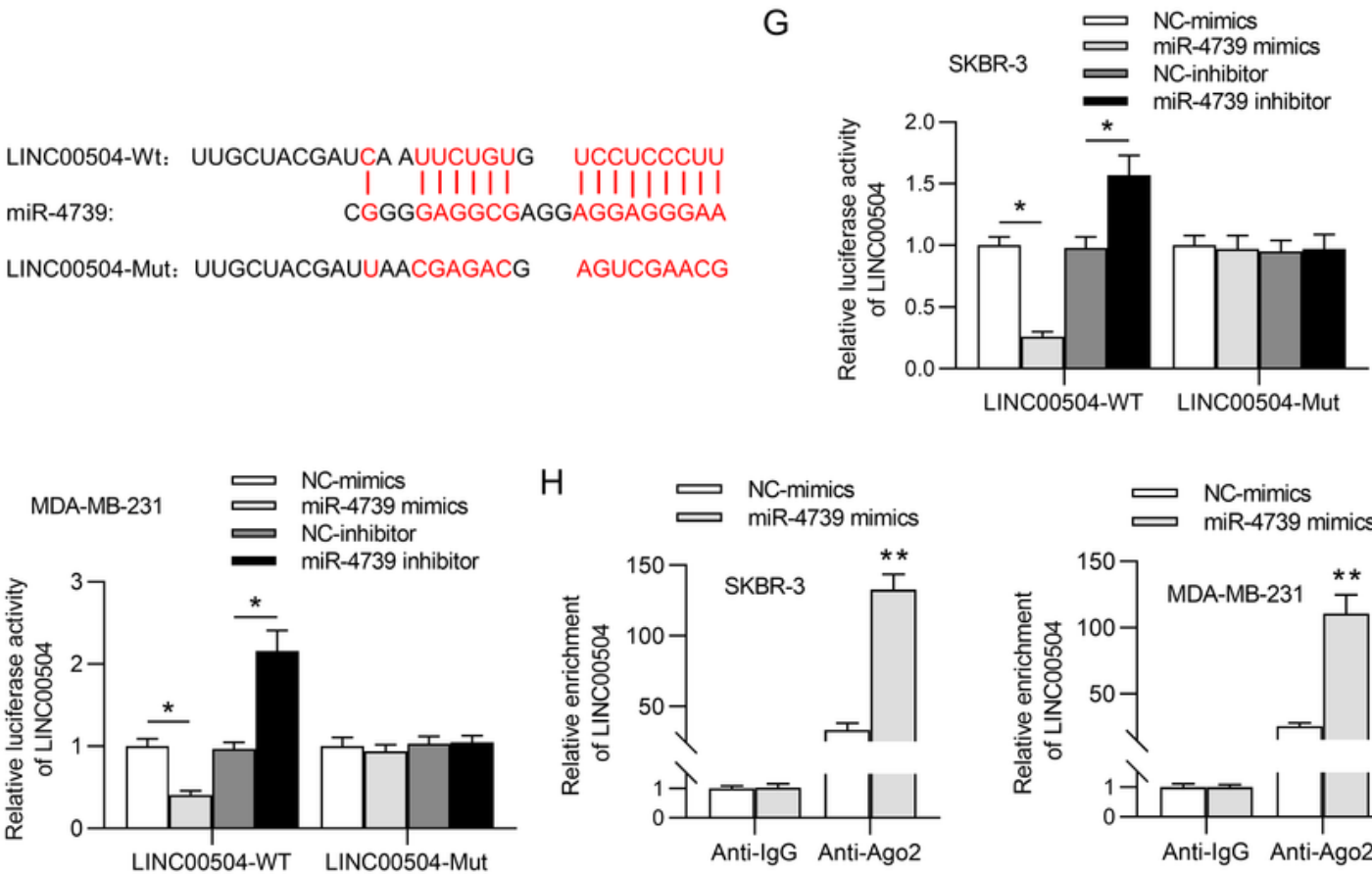

H

NC-mimics

miR-4739 mimics
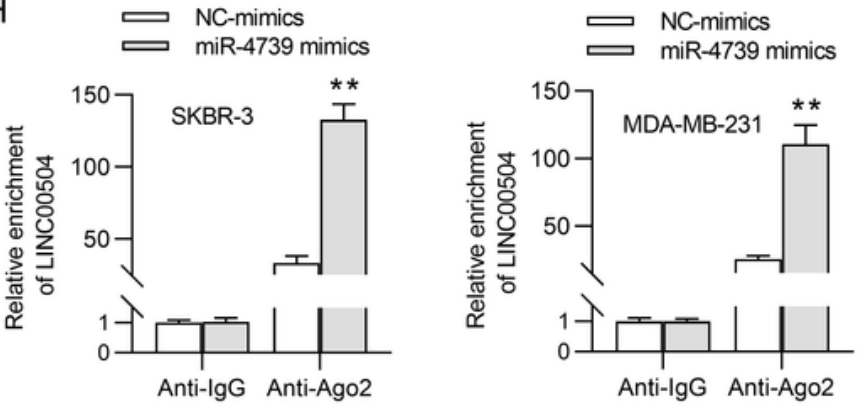

Figure 4

LINC00504 serves as a molecular sponge of miR-4739. (A) Subcellular fraction assay disclosed the location and distribution of LINC00504 in the cytoplasm and nucleus in TNBC cells. (B) RT-qPCR detected the relative expression of 5 potential miRNAs in TNBC cells. (C) The miR-4739 expression in TNBC tissues and adjacent normal tissues was detected. (D) RT-qPCR measured the relative expression of miR-4739 in NC-mimics, miR-4739 mimics group. (E) RT-qPCR determined the relative expression of miR-4739 in NC- 
inhibitor or miR-4739 inhibitor group. (F) The binding site between miR-4739 and LINC00504 was predicted through starBase v2.0. (G) Luciferase report assay revealed luciferase activity of LINC00504 by miR-4739 mimics or miR-4739 inhibitor. (H) RIP assay revealed relative enrichment of LINC00504 precipitated by anti-IgG and anti-Ago2 in NC-mimics and miR-4739 mimics group. ${ }^{*} \mathrm{p} \otimes 0.05,{ }^{* *} \mathrm{p}<0.01$.

A

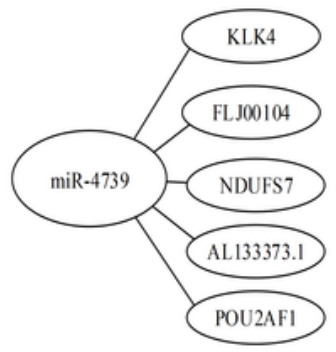

B

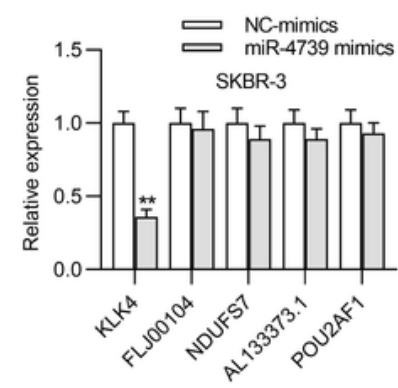

$\mathrm{E}$
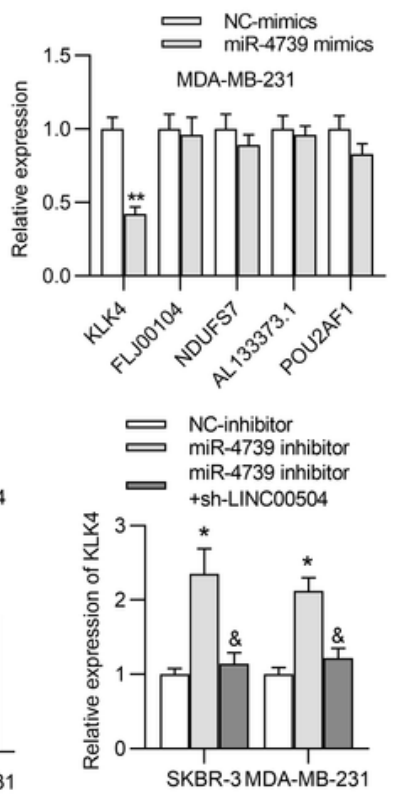

C
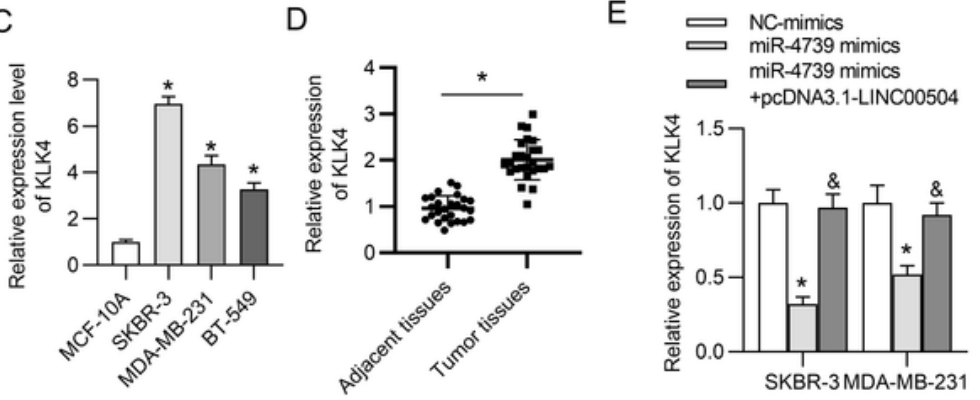

Anti-lgG

G

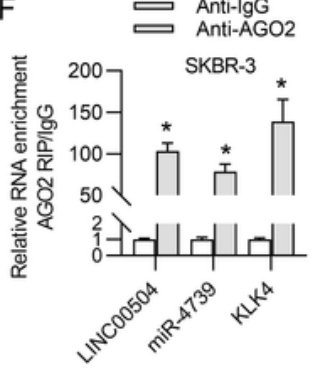

$\mathrm{H}$

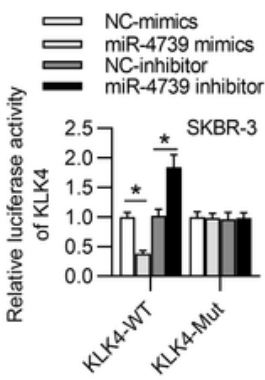

$J$

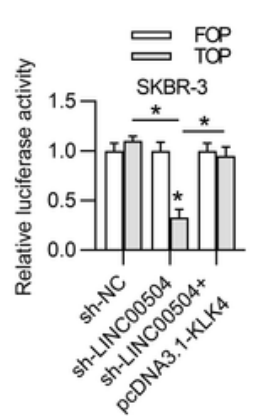

KLK4-Wt: 5' AGGCCCCCAGCCCCU - CCUCCCUC

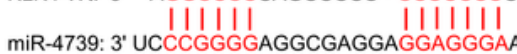
KLK4-Mut: 5' ACCUGAUCAGCCCCU - GACUUACC
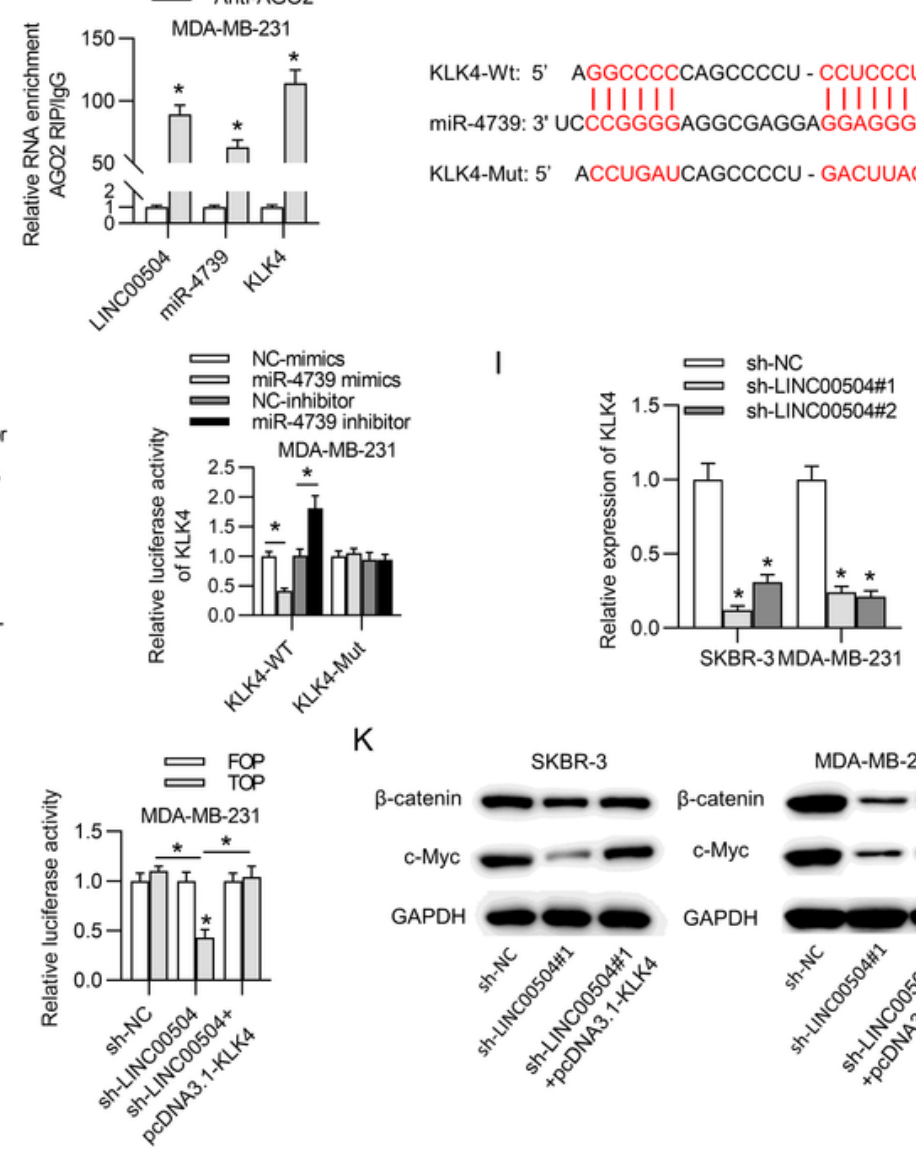

$\mathrm{K}$

SKBR-3

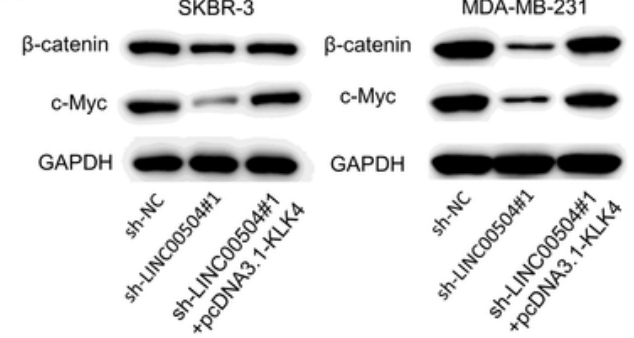

Figure 5 
KLK4 is the downstream target of miR-4739. (A) TargetScan website revealed 5 potential target genes of miR-4739. (B) RT-qPCR measured the expressions of five candidate mRNAs after upregulating miR-4739. (C-D) RT-qPCR determined KLK4 expression in TNBC cells and tissues. (E) Relative expression levels of KLK4 in SKBR-3 and MDA-MB-231 cells after transfection with miR-4739 mimics, miR-4739 mimics+pcDNA3.1-LINC00504, miR-4739 inhibitor, miR-4739 inhibitor+pcDNA3.1-LINC00504 were measured by RT-qPCR. (F) RIP assay revealed the relative enrichment of LINC00504, miR-4739 and KLK4. (G) The binding site between miR-4739 and 3'UTR of KLK4 was predicted by TargetScan. (H) Luciferase report assay validated the direct interaction among LINC00504, miR-4739 and KLK4. (I) Knockdown efficiency of KLK4 was verified through RT-qPCR. (J) TOP/FOP flash assay was used to verify the activity of the Wnt/ $\beta$-catenin pathway. $(K)$ Western blot showed the rescue effects of KLK4 on the inactivated Wnt/ $\beta$-catenin pathway caused by silenced LINC00504. Full-length blots are presented in Supplementary material. The ImageJ software was used to detect densitometry analysis of the band intensity. ${ }^{\star} \mathrm{p} \otimes 0.05$, **p $₫ 0.01$. 
A

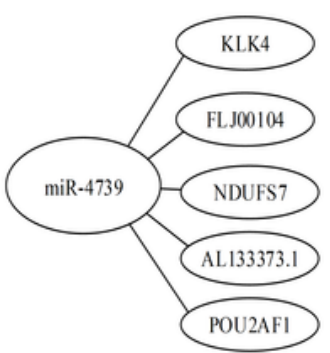

B

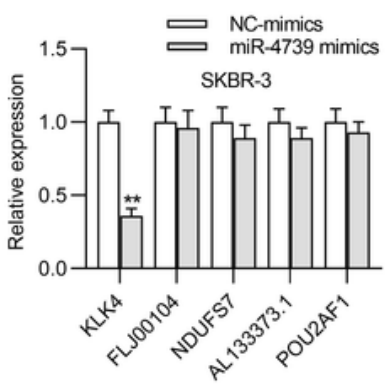

E

NC-mimics

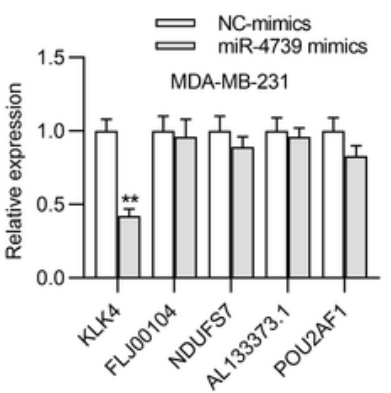

C

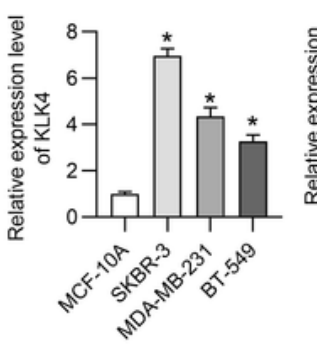

D
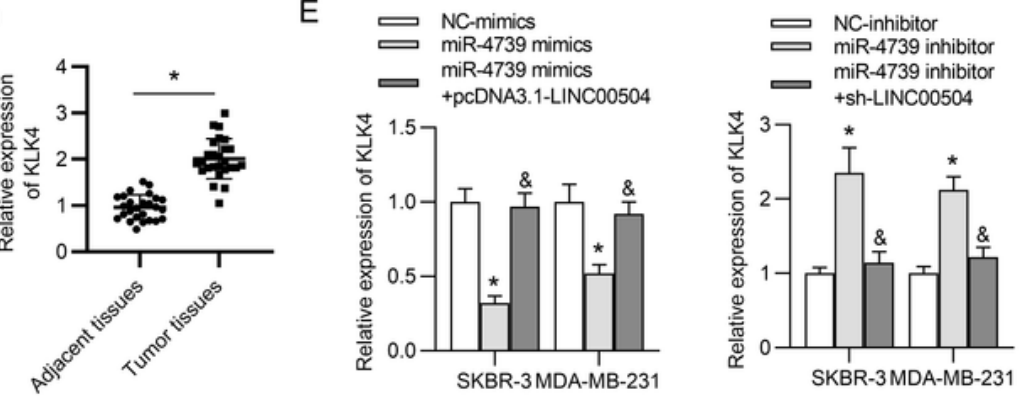

F
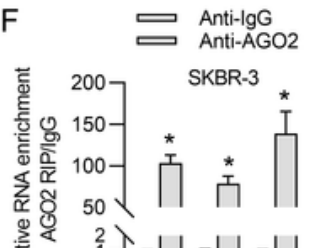

迹 ${ }_{0}^{2}$ 대메대

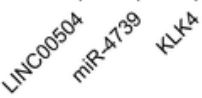

H
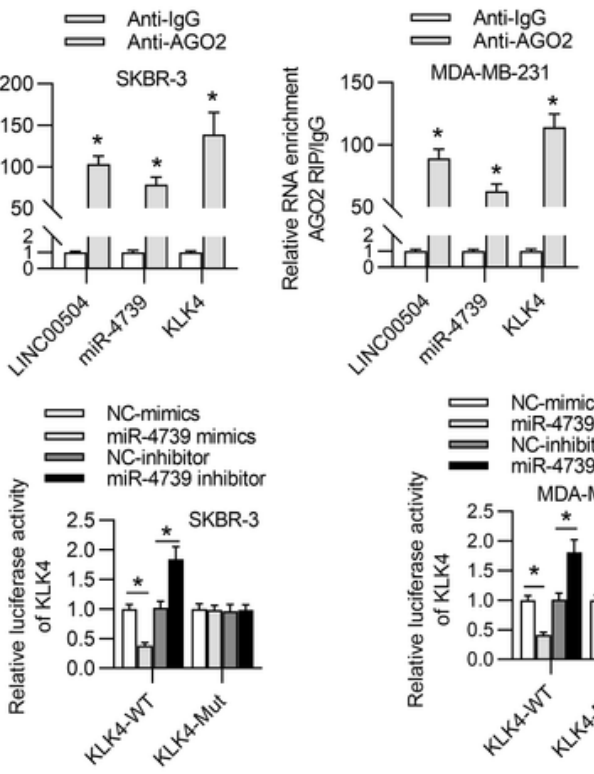

G

KLK4-Wt: 5' AGGCCCCCCAGCCCCU - CCUCCCUC miR-4739: 3' UCCCGGGGAGGCGAGGAGGAGGGAA KLK4-Mut: 5' ACCUGAUCAGCCCCU - GACUUACC

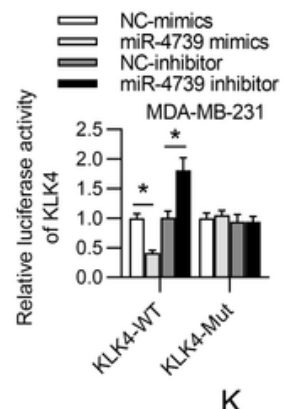

J
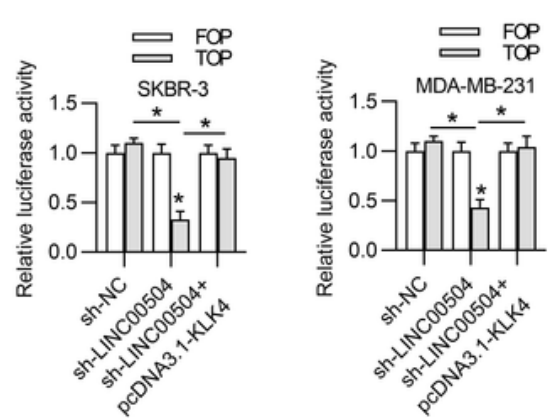

K

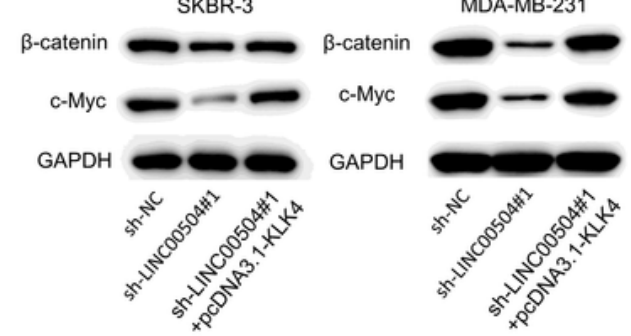

Figure 5

KLK4 is the downstream target of miR-4739. (A) TargetScan website revealed 5 potential target genes of miR-4739. (B) RT-qPCR measured the expressions of five candidate mRNAs after upregulating miR-4739. (C-D) RT-qPCR determined KLK4 expression in TNBC cells and tissues. (E) Relative expression levels of KLK4 in SKBR-3 and MDA-MB-231 cells after transfection with miR-4739 mimics, miR-4739 mimics+pcDNA3.1-LINC00504, miR-4739 inhibitor, miR-4739 inhibitor+pcDNA3.1-LINC00504 were 
measured by RT-qPCR. (F) RIP assay revealed the relative enrichment of LINC00504, miR-4739 and KLK4. (G) The binding site between miR-4739 and 3'UTR of KLK4 was predicted by TargetScan. (H) Luciferase report assay validated the direct interaction among LINC00504, miR-4739 and KLK4. (I) Knockdown efficiency of KLK4 was verified through RT-qPCR. (J) TOP/FOP flash assay was used to verify the activity of the Wnt/ $\beta$-catenin pathway. $(K)$ Western blot showed the rescue effects of KLK4 on the inactivated Wnt/ $\beta$-catenin pathway caused by silenced LINC00504. Full-length blots are presented in Supplementary material. The ImageJ software was used to detect densitometry analysis of the band intensity. ${ }^{*} \mathrm{p} \otimes 0.05$, $\star * \mathrm{p} \otimes 0.01$. 


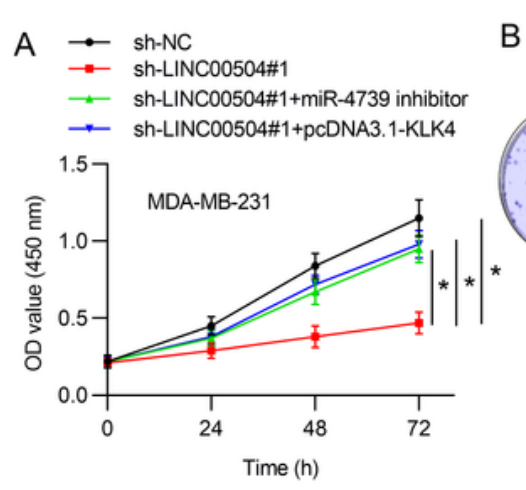

C
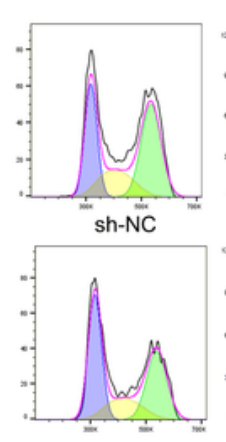
4739 inhibitor
B

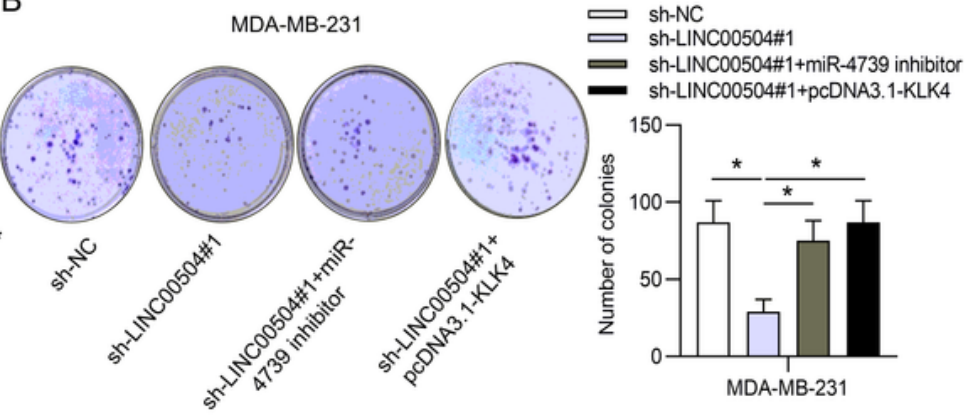

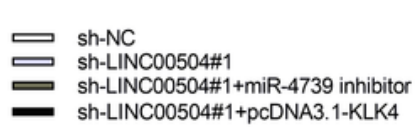

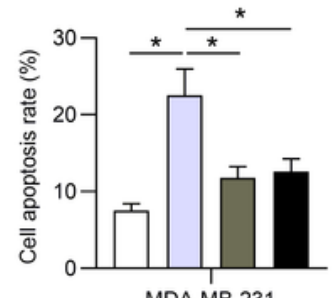

MDA-MB-231

F

sh-NC

sh-LINCO0504\#1

sh-LINC00504\#1+miR-4739 inhibitor

- sh-LINC00504\#1+pcDNA3.1-KLK4

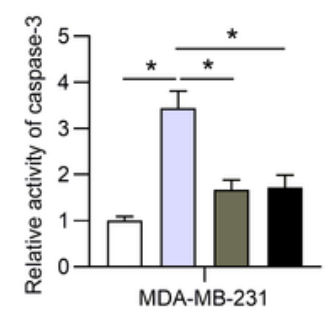

D MDA-MB-231
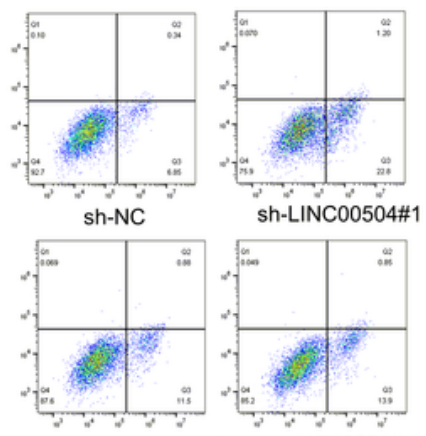

sh-LINC00504\#1+miR- sh-LINC00504\#1+ 4739 inhibitor pcDNA3.1-KLK4

E
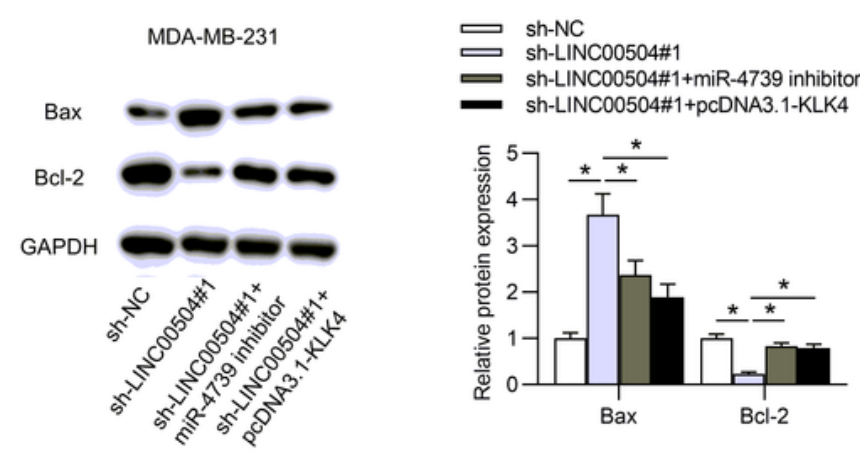

\section{Figure 6}

The LINC00504/miR-4739/KLK4 axis promotes cell proliferation, cell cycle progression, and suppresses cell apoptosis by activating the Wnt/ $\beta$-catenin pathway in TNBC. (A) Cell viability of MDA-MB-231 cells was detected by CCK-8 assays in 4 groups: sh-NC, sh-LINC00504\#1, sh-LINC00504\#1+miR-4739 inhibitor, sh-LINC00504\#1+ pcDNA3.1-KLK4. (B) Colony formation assay was applied to evaluate the colony numbers. (C) Flow cytometry detected the cell cycle progression. (D) Flow cytometry analysis showed the 
rate of cell apoptosis. (E) Western blot determined protein expressions of Bax and Bcl-2. Full-length blots are presented in Supplementary material. The ImageJ software was used to detect densitometry analysis of the band intensity. (F) Caspase-3 Colorimetric Assay Kit measured the activity of caspase-3. *pख0.05.

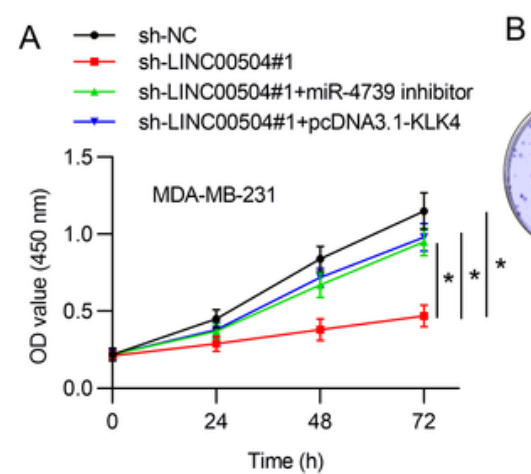

C
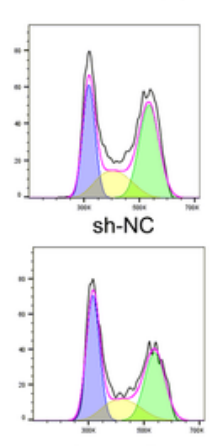

Sh-LINC00504\#1+m 4739 inhibitor
B MDA-MB-231

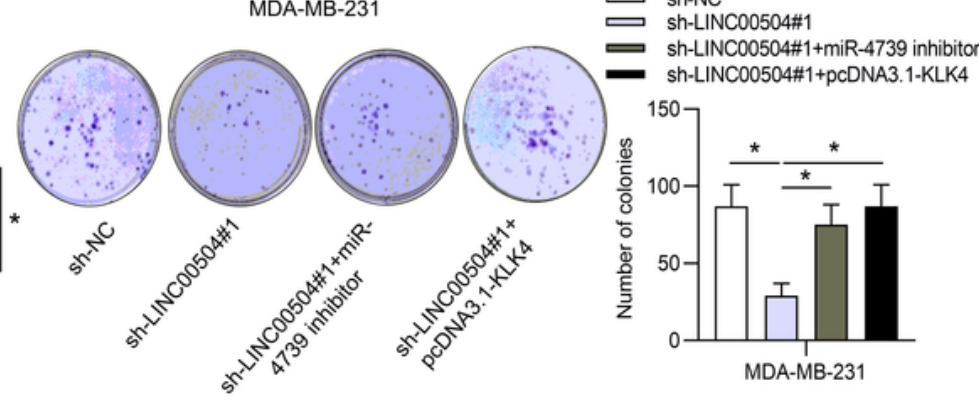

D

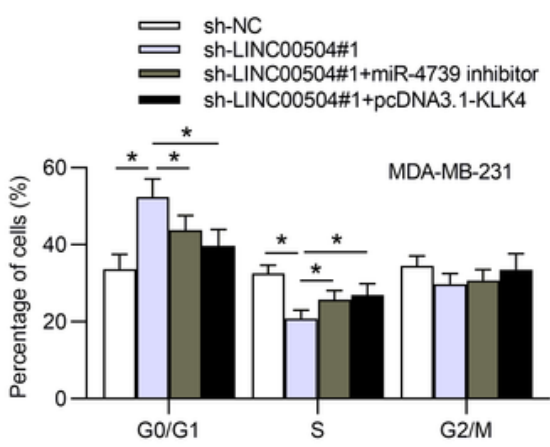

E

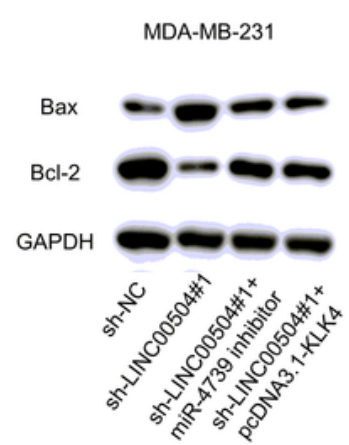

MDA-MB-231

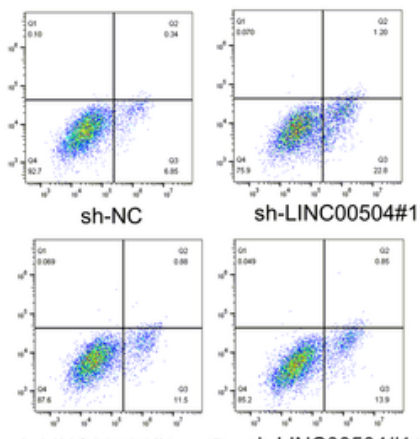
sh-LINC00504\#1+miR-
4739 inhibitor
pCDNA3

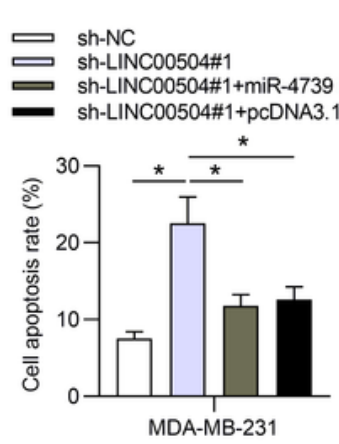

F

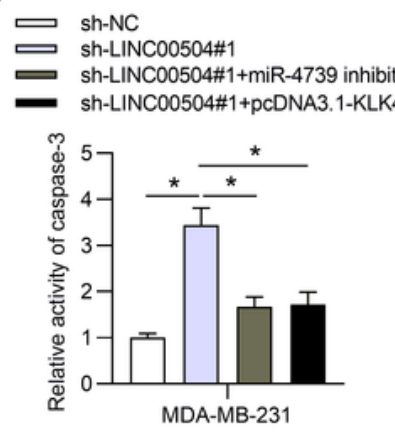

Figure 6

The LINC00504/miR-4739/KLK4 axis promotes cell proliferation, cell cycle progression, and suppresses cell apoptosis by activating the Wnt/ $\beta$-catenin pathway in TNBC. (A) Cell viability of MDA-MB-231 cells 
was detected by CCK-8 assays in 4 groups: sh-NC, sh-LINC00504\#1, sh-LINC00504\#1+miR-4739 inhibitor, sh-LINC00504\#1+ pcDNA3.1-KLK4. (B) Colony formation assay was applied to evaluate the colony numbers. (C) Flow cytometry detected the cell cycle progression. (D) Flow cytometry analysis showed the rate of cell apoptosis. (E) Western blot determined protein expressions of Bax and Bcl-2. Full-length blots are presented in Supplementary material. The Image J software was used to detect densitometry analysis of the band intensity. (F) Caspase-3 Colorimetric Assay Kit measured the activity of caspase-3. *pख0.05.

\section{Supplementary Files}

This is a list of supplementary files associated with this preprint. Click to download.

- Supplementarymaterialfig.2G.TIF

- Supplementarymaterialfig.2G.TIF

- Supplementarymaterialfig.5K.TIF

- Supplementarymaterialfig.5K.TIF

- Supplementarymaterialfig.6E.TIF

- Supplementarymaterialfig.6E.TIF 